Facundo Mémoli

and

Guillermo Sapiro

IMA Preprint Series \# 1980

( June 2004)

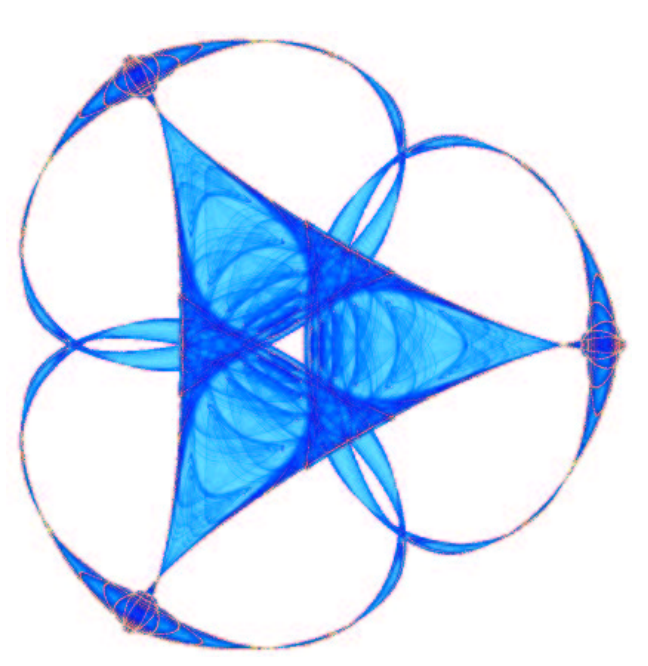

INSTITUTE FOR MATHEMATICS AND ITS APPLICATIONS

UNIVERSITY OF MINNESOTA

514 Vincent Hall

206 Church Street S.E.

Minneapolis, Minnesota 55455-0436

Phone: 612/624-6066 Fax: 612/626-7370

URL: http://www.ima.umn.edu 


\title{
A Theoretical and Computational Framework for Isometry Invariant Recognition of Point Cloud Data
}

\author{
Facundo Mémoli* Guillermo Sapiro ${ }^{\dagger}$
}

\begin{abstract}
Point clouds are one of the most primitive and fundamental manifold representations. A popular source of point clouds are three dimensional shape acquisition devices such as laser range scanners. Another important field where point clouds are found is in the representation of highdimensional manifolds by samples. With the increasing popularity and very broad applications of this source of data, it is natural and important to work directly with this representation, without having to go through the intermediate and sometimes impossible and distorting steps of surface reconstruction. A geometric framework for comparing manifolds given by point clouds is presented in this paper. The underlying theory is based on Gromov-Hausdorff distances, leading to isometry invariant and completely geometric comparisons. This theory is embedded in a probabilistic setting as derived from random sampling of manifolds, and then combined with results on matrices of pairwise geodesic distances leading to a computational implementation of the framework. The theoretical and computational results here presented are complemented with experiments for real three dimensional shapes.
\end{abstract}

${ }^{*}$ Electrical and Computer Engineering, University of Minnesota, Minneapolis, MN 55455; and Instituto de Ingeniería Eléctrica, Universidad de la República, Montevideo, Uruguay, memoli@ece.umn.edu.

${ }^{\dagger}$ Electrical and Computer Engineering and Digital Technology Center, University of Minnesota, Minneapolis, MN 55455, guille@ece.umn.edu 


\section{Introduction}

Point clouds are one of the most primitive and fundamental manifold representations. One of the most popular sources of point clouds are $3 D$ shape $^{1}$ acquisition devices, such as laser range scanners, with applications in geoscience, art (e.g., archival), medicine (e.g., prohestetics), manufacturing (from cars to clothes), and security (e.g., face recognition), among other disciplines. These scanners in general provide raw data in the form of (maybe noisy) unorganized point clouds representing surface samples. With the increasing popularity and very broad applications of this source of data, it is natural and important to work directly with this representation, without having to go to the intermediate step of fitting a surface to it (step that can add computational complexity and introduce errors). See for example $[5,14,17,20,26,36,37,44,45]$ for a few of the recent works with this type of data. Point clouds can also be used as primitives for visualization, e.g., [6, 26, 48], as well as for editing [52].

Another important field where point clouds are found is in the representation of high-dimensional manifolds by samples (see for example [2, 30, 35, 49]). This type of high-dimensional and general co-dimension data appears in almost all disciplines, from computational biology to image analysis to financial data. Due to the extremely high dimensionality in this case, it is impossible to perform manifold reconstruction, and the tasks need to be performed directly on the raw data, meaning the point cloud.

The importance of this type of shape representation is leading to an increase in the fundamental study of point clouds, from its basic geometric characteristics such as curvature [40] and intrinsic distances and geodesics [39], to intrinsic dimensionality and topological structure [2, 12, 15, 16, 21, 49], and also including the detection of particular structures [1] (see also the papers mentioned in the first paragraph and references therein). The goal of this work, inspired in part by [18] and the tools developed in [39, 49], is to develop a theoretical and computational framework to compare shapes represented as point clouds. We are then going to assume the existence of an underlying structure, which belongs to a certain class of objects, from which our point cloud data are sampled.

As we have mentioned, a variety of objects can be represented as point clouds in $\mathbb{R}^{d}$. One is often presented with the problem of deciding whether two of those point clouds, and/or their corresponding underlying objects or manifolds, represent the same geometric structure or not (object recognition and classification). We are then concerned with questions about the underlying unknown structures (objects), which need to be answered based on discrete measures taken between their respective point clouds. In greater generality, we may wonder what is the structural information we can gather about the object itself by exploring a point cloud which represents it. ${ }^{2}$

Multidimensional scaling (MDS), for example, has been used to partly approach this general problem of object analysis/recognition, by means of checking whether the underlying space (object) is flat or not, and also providing information about the object's dimensionality (as a subset of $\mathbb{R}^{d}$ ) and its projection into a reduced space. Procedures based on MDS require that one first computes the interpoint distance matrix for all the members of the point cloud (or for a representative selected sub-set of them). If one is interested in comparing two different objects, the problem is reduced to a comparison between the corresponding interpoint distance matrices of their point clouds. If the distance we use is the Euclidean one, these matrices only provide information about their rigid similarity, and (assuming the matrices are of the same size) if they are equal (up to permutations

\footnotetext{
${ }^{1}$ We will loosely use the terms shape/object/surface in this introduction to refer to either surfaces in space or Riemannian manifolds in more generality. A more precise notion will be given later on.

${ }^{2} \mathrm{~A}$ related important question is what conditions must a point verify in order to faithfully represent an object, not to mention that one must define faithfully.
} 
of the indices of all elements), ${ }^{3}$ we can only conclude that there exists a rigid isometry (rotation, reflection, translation) from one point cloud to the other. Under assumptions of compactness we can also say something about the true underlying objects. Let's be more precise. Let the point clouds $\mathcal{P}_{i} \subset S_{i}$ be $\epsilon_{i}$-coverings of the compact surfaces $S_{i}$ in $\mathbb{R}^{3}$, for $i=1,2$ (this will be formally defined below). Then assuming there exists a rigid isometry $\tau: \mathbb{R}^{3} \rightarrow \mathbb{R}^{3}$ such that $\tau\left(\mathcal{P}_{1}\right)=\mathcal{P}_{2}$, we can bound the Hausdorff distance (which we will also formally define below) between $\tau\left(S_{1}\right)$ and $S_{2}$ as follows:

$$
\begin{aligned}
d_{\mathcal{H}}\left(\tau\left(S_{1}\right), S_{2}\right) & \leq d_{\mathcal{H}}\left(\tau\left(S_{1}\right), \tau\left(\mathcal{P}_{1}\right)\right)+d_{\mathcal{H}}\left(\tau\left(\mathcal{P}_{1}\right), \mathcal{P}_{2}\right)+d_{\mathcal{H}}\left(\mathcal{P}_{2}, S_{2}\right) \\
& =d_{\mathcal{H}}\left(S_{1}, \mathcal{P}_{1}\right)+d_{\mathcal{H}}\left(\tau\left(\mathcal{P}_{1}\right), \mathcal{P}_{2}\right)+d_{\mathcal{H}}\left(\mathcal{P}_{2}, S_{2}\right) \\
& \leq \epsilon_{1}+0+\epsilon_{2}
\end{aligned}
$$

And of course the same kind of bound holds for the Hausdorff distance between the point clouds once we assume the underlying continuous objects are rigidly isometric, see $\S 2.1$ below.

One possible modification would be considering, still for compact surfaces, the intrinsic distance instead the Euclidean (extrinsic) one for the construction of the aforementioned interpoint distance matrices. A comparison of these new distance matrices would then allow for more freedom in deciding when two objects are similar since now bends are allowed.

If $S_{1}$ and $S_{2}$ happen to be isometric (here also allowing for bends and not only rigid transformations) we wonder whether we will be able to detect this by looking at (finite) point clouds $\mathcal{P}_{i}$ sampled from each $S_{i}$. This problem is harder to tackle. We approach it through a probabilistic model, since in principle there might exist even for the same object, two different samplings that look quite dissimilar (under discrete measures we can cope with computationally), for arbitrarily fine scales (see below).

With the help of the theory here presented we recast these considerations in a rigorous framework and address the case where the distances considered to characterize each point cloud (object) are more general. We concentrate on the case when there exists an intrinsic notion of distance for each object we sample. For the applications of isometry invariant shape (surfaces) recognition, one must therefore consider the distance as measured by paths constrained to travel on the surface of the objects, better referred to as geodesic distance. These ideas of using geodesic distances have been introduced and used in $[8,18]$ for bending invariant recognition in $3 D$, e.g., articulated objects (the theoretical foundations here introduced provide a justification of such approach), and in [21, 49] to detect intrinsic surface dimensionality.

In this paper we introduce both a theoretical and computational framework for the so called isometry invariant shape recognition problem. The theory we use and build our framework upon is that pioneered by Gromov [24], in which a metric is introduced in the space of all (compact) metric spaces. For the sake of generality we present most of the framework for metric spaces, but the reader, at any moment, is invited to think of surfaces for simplicity. We will abuse terminology in the following sense: Since we are dealing both with metric spaces and finite sets of samples from them, we are going to speak of continuous and discrete metric spaces. For instance, given a metric space $(X, d)$ we consider a finite subset of it, $\mathbb{X}_{m} \subset X$ which we endow with the metric of $X$ to conform a discrete metric space, then $X$ will be called continuous. This is in analogy with the sampling of signals.

\footnotetext{
${ }^{3}$ Boutin and Kemper, [7], have approached the recognition problem for (discrete objects) by looking only at the histogram of interpoint squared Euclidean distances. Interestingly, they have concluded that while there are counterexamples for the recognition problem with this kind of representation, they constitute a very small fraction of all the possible point configurations.
} 
The fundamental approach used for isometry invariant recognition in this paper is derived then from the Gromov-Hausdorff distance, which we now proceed to present. Suppose $X$ and $Y$ are two (objects) compact subsets of a common bigger metric space $(Z, d)$, and we want to compare $X$ to $Y$ in order to decide whether they are/represent the same object or not. Then, an idea that one might come up with very early on is that of computing the Hausdorff distance between them (see for example $[13,28]$ for an extensive use of this for shape statistics and image comparison):

$$
d_{\mathcal{H}}^{Z}(X, Y):=\max \left(\sup _{x \in X} d(x, Y), \sup _{y \in Y} d(y, X)\right)
$$

But, what happens if we want to allow for certain deformations to occur and still decide that the objects are the same? More precisely, we are interested in being able to find a distance between metric spaces that is intrinsic to the object and blind to isometric transformations ("bends"). This will permit a truly geometric comparison between the manifolds, independently of their embedding and bending position. Following [24], we introduce the Gromov-Hausdorff distance between Metric Spaces:

$$
d_{\mathcal{G H}}(X, Y):=\inf _{Z, f, g} d_{\mathcal{H}}^{Z}(X, Y)
$$

where $f: X \rightarrow Z$ and $g: Y \rightarrow Z$ are isometric embeddings (distance preserving) into the metric space $Z$. It turns out that this measure of metric proximity between metric spaces is well suited for our problem at hand and will allow us to give a formal framework to address the isometric shape recognition problem (for point cloud data). However, this notion of distance between metric spaces encodes the "metric" disparity between them, at first glance, in a computationally impractical way. We derive below new results that connect this notion of disparity with other more computationally appealing expressions.

We have in mind specific applications and scenarios such as those described above, and in particular surfaces and submanifolds of some Euclidean space $\mathbb{R}^{d}$. We assume that we are given as input points densely sampled from the metric spaces (surfaces, manifolds). This will manifest itself in many places in the theory described below. We will present a way of computing a discrete approximation (or bound) to $d_{\mathcal{G H}}($,$) based on the metric information provided by these point$ clouds.

The problem of isometry invariant shape recognition at hand can be split in two parts. Firstly, suppose the metric spaces under consideration happen to be isometric. We then have to guarantee that we can discover this by looking at a computable discrete measure of metric similarity based just on our observed data, that is, the point clouds. Secondly, if that measure of (discrete) metric similarity is "small," what can we say about that metric similarity between the underlying metric spaces? Both parts are addressed in our work. One cannot perform object recognition without either of them.

The rest of this paper is organized as follows: The basic theoretical foundations are given in Section $\S 2$, Section $\S 3$ presents the computational foundations, Section $\S 4$ confirms the theory with real examples, and finally Section $\S 5$ concludes the paper and describes current efforts and future directions.

\section{$2 \quad$ Theoretical Foundations}

This section covers the fundamental theory behind the bending invariant recognition framework we develop. We first introduce some basic notation, definitions, and classical results. We use basic 
concepts of metric spaces, see for example [31] for a simple exposition of this.

Definition 1 (Metric Space) $A$ set $M$ is a metric space if for every pair of points $x, y \in M$ there is a well defined function $d_{M}(x, y)$ whose values are non-negative real numbers, such that (a) $d_{M}(x, y)=0 \Leftrightarrow x=y$, and (b) $d_{M}(x, y) \leq d_{M}(y, z)+d_{M}(z, x)$ for any $x, y$ and $z \in M$. We call $d_{M}: M \times M \rightarrow \mathbb{R}$ the metric or distance. For clarity we will specify a metric space as the pair $\left(M, d_{M}\right)$.

Definition 2 (Covering) For a point $x$ in the metric space $\left(X, d_{X}\right)$ and $r>0$, we will denote by $B_{X}(x, r)$ the set $\left\{z \in X \mid d_{X}(x, z)<r\right\}$. For a subset $A$ of $X$, we use the notation $B_{X}(A, r)=$ $\cup_{a \in A} B_{X}(a, r)$. We say that a set $C \subset X$ is an $R$-covering of $X$ if $B_{X}(C, R)=X$. We will also frequently say that the set $A$ is a n-covering of $X$ if $A$ constitutes, for some $r>0$, a covering of $X$ by $n$-balls with centers in points of $A$.

Definition 3 (Isometry) We say the metric spaces $\left(X, d_{X}\right)$ and $\left(Y, d_{Y}\right)$ are isometric when there exists a bijective mapping $\phi: X \rightarrow Y$ such that $d_{X}\left(x_{1}, x_{2}\right)=d_{Y}\left(\phi\left(x_{1}\right), \phi\left(x_{2}\right)\right)$ for all $x_{1}, x_{2} \in X$. Such $a \phi$ is an isometry between $\left(X, d_{X}\right)$ and $\left(Y, d_{Y}\right)$.

Next, we state some well known properties of the Gromov-Hausdorff distance $d_{\mathcal{G H}}($,$) which will$ be useful for our presentation.

Proposition 1 1. Let $\left(X, d_{X}\right),\left(Y, d_{Y}\right)$ and $\left(Z, d_{Z}\right)$ be metric spaces then

$$
d_{\mathcal{G H}}(X, Y) \leq d_{\mathcal{G H}}(X, Z)+d_{\mathcal{G H}}(Z, Y) .
$$

2. If $d_{\mathcal{G H}}(X, Y)=0$ and $\left(X, d_{X}\right),\left(Y, d_{Y}\right)$ are compact metric spaces, then $\left(X, d_{X}\right)$ and $\left(Y, d_{Y}\right)$ are isometric.

3. Let $\left\{x_{1}, \ldots, x_{n}\right\} \subset X$ be a $R$-covering of the compact metric space $\left(X, d_{X}\right)$.

Then $d_{\mathcal{G H}}\left(X,\left\{x_{1}, \ldots, x_{n}\right\}\right) \leq R$.

4. For compact metric spaces $\left(X, d_{X}\right)$ and $\left(Y, d_{Y}\right)$ :

$$
\frac{1}{2}|\operatorname{rad}(X)-\operatorname{rad}(Y)| \leq d_{\mathcal{G H}}(X, Y) \leq \frac{1}{2} \max (\operatorname{diam}(X), \operatorname{diam}(Y))
$$

where $\operatorname{rad}(X):=\min _{x \in X} \max _{x^{\prime} \in X} d_{X}\left(x, x^{\prime}\right)$ and $\operatorname{diam}(X):=\max _{x, x^{\prime} \in X} d_{X}\left(x, x^{\prime}\right)$ stand for the circumradius and diameter of the metric space $\left(X, d_{X}\right)$, respectively.

5. For bounded metric spaces $\left(X, d_{X}\right)$ and $\left(Y, d_{Y}\right)$,

$$
d_{\mathcal{G H}}(X, Y)=\inf _{\substack{\phi: X \rightarrow Y \\ \psi: Y \rightarrow X}} \sup _{x \in X, y \in Y} \frac{1}{2}\left|d_{X}(x, \psi(y))-d_{Y}(y, \phi(x))\right|
$$

The proofs of properties 1 to 4 can be gleaned from $[9,24,27,46]$, and property 5 can be found in [32]. Also of great informative value is [47].

Remark 1 Since for compact $X, \operatorname{rad}(X) \in\left[\frac{\operatorname{diam}(X)}{2}, \operatorname{diam}(X)\right]$, from property 4 it follows that two metric spaces whose diameters differ a lot must be at a positive $d_{\mathcal{G H}}($,$) distance, as intuition$ requires. 
From these properties, we can also easily obtain the following important result:

Corollary 1 Let $X$ and $Y$ be compact metric spaces. Let moreover $\mathbb{X}_{m}$ be a r-covering of $X$ (consisting of $m$ points) and $\mathbb{Y}_{m^{\prime}}$ be a $r^{\prime}$-covering of $Y$ (consisting of $m^{\prime}$ points). Then

$$
\left|d_{\mathcal{G H}}(X, Y)-d_{\mathcal{G H}}\left(\mathbb{X}_{m}, \mathbb{Y}_{m^{\prime}}\right)\right| \leq r+r^{\prime}
$$

We can then say that if we could compute $d_{\mathcal{G H}}($,$) for discrete metric spaces which are dense$ enough samplings of the "continuous" underlying ones, that number would be a good approximation to what happens between the continuous spaces. Currently, there is no computationally efficient way to directly compute $d_{\mathcal{G H}}($,$) between discrete metric spaces in general. This forces us$ to develop a roundabout path, see $\S 2.2$ ahead. Before going into the general case, we discuss next the application of the ideas of our framework to a simpler but important case.

\subsection{Intermezzo: The Case of Rigid Isometries}

When we try to compare two (compact) subsets $X$ and $Y$ of a larger metric space $Z$, the situation is a bit simpler. The measure of similarity boils down to a somewhat simpler Hausdorff distance between the sets (which of course must take into account self-isometries of $Z$ ). In more detail, one must compute

$$
d_{\mathcal{H}}^{Z, \text { rigid }}(X, Y):=\inf _{\Phi} d_{\mathcal{H}}^{Z}(X, \phi(Y))
$$

where $\Phi: Z \rightarrow Z$ ranges over all self-isometries of $Z$. If we knew an efficient way of computing $\inf _{\Phi} d_{\mathcal{H}}^{Z}(X, \Phi(Y))$, then this restricted shape recognition problem would be well posed for $Z$, in view of an adapted version of Proposition 1 and Corollary 1, as soon as we can give guarantees of coverage. For the sake of completeness we state such a result.

Proposition $2 d_{\mathcal{H}}^{Z, r i g i d}(\cdot, \cdot)$ satisfies the triangle inequality and in particular, the following relation holds:

$$
\left|d_{\mathcal{H}}^{Z, \text { rigid }}(X, Y)-d_{\mathcal{H}}^{Z, r i g i d}\left(\mathbb{X}_{m}, \mathbb{Y}_{m^{\prime}}\right)\right| \leq r+r^{\prime}
$$

for compact $X, Y \subset Z$ such that $X \subset B_{Z}\left(\mathbb{X}_{m}, r\right)$ and $Y \subset B_{Z}\left(\mathbb{Y}_{m^{\prime}}, r^{\prime}\right)$.

Coverage can be guaranteed, in the case of submanifolds of $\mathbb{R}^{d}$, by imposing a probabilistic model on the samplings $\mathbb{X}_{m}$ of the manifolds, and a bound on the curvatures of the family of manifolds one wishes to work with. In more detail, we can show that

$$
\mathbf{P}\left(d_{\mathcal{H}}^{\mathbb{R}^{d}}\left(X, \mathbb{X}_{m}\right)>\delta_{m}\right) \simeq \frac{1}{\ln m} \text { as } m \uparrow \infty
$$

for $\delta_{m} \gtrsim\left(\frac{\ln m}{m}\right)^{1 / k}$, where $k$ is the dimension of $X$, see Section $\S 3.3$.

In the case of surfaces in $Z=\mathbb{R}^{3}, \Phi$ sweeps all rigid isometries, and there exist good algorithms which can actually solve the problem approximately. For example, in [22] the authors report an algorithm which for any given $0<\alpha<1$ can find a rigid transformation $\widehat{\Phi}_{\alpha}$ such that

$$
d_{\mathcal{H}}^{\mathbb{R}^{3}}\left(\mathbb{X}_{m}, \widehat{\Phi}_{\alpha}\left(\mathbb{Y}_{m^{\prime}}\right)\right) \leq(8+\alpha) \inf _{\Phi} d_{\mathcal{H}}^{\mathbb{R}^{3}}\left(\mathbb{X}_{m}, \Phi\left(\mathbb{Y}_{m^{\prime}}\right)\right)
$$


with complexity $O\left(s^{4} \log s\right)$ where $s=\max \left(m, m^{\prime}\right)$. This computational result, together with simple considerations, makes the problem of surface recognition (under rigid motions) well posed and well justified. In fact, using Proposition 2 we obtain a bound between the distance we want to estimate $d_{\mathcal{H}}^{\mathbb{R}^{3}, \text { rigid }}(X, Y)$ and the observable (computable) value $d_{\mathcal{H}}^{\mathbb{R}^{3}}\left(\mathbb{X}_{m}, \widehat{\Phi}_{\alpha}\left(\mathbb{Y}_{m^{\prime}}\right)\right)$ :

$$
d_{\mathcal{H}}^{R^{3}, \text { rigid }}(X, Y)-\left(r+r^{\prime}\right) \leq d_{\mathcal{H}}^{\mathbb{R}^{3}}\left(\mathbb{X}_{m}, \widehat{\Phi}_{\alpha}\left(\mathbb{Y}_{m^{\prime}}\right)\right) \leq 10\left(d_{\mathcal{H}}^{\mathbb{R}^{3}, \text { rigid }}(X, Y)+\left(r+r^{\prime}\right)\right)
$$

Equation (2) gives a formal justification to the procedure outlined for this surface recognition problem. To the best of our knowledge, this is the first time such formality is presented for this very important problem, both in the particular case just shown and for the general one addressed next. In any case, if $d_{\mathfrak{S}}$ is the measure of similarity between the continuous objects we are considering, and $\widehat{d_{\mathcal{S}}}$ is the computable approximate measure of similarity between the discrete samples, the kind of relation we seek to establish is

$$
A\left(d_{\mathcal{S}}(X, Y)-\alpha\right) \leq \widehat{d_{\mathcal{S}}}\left(\mathbb{X}_{m}, \mathbb{Y}_{m^{\prime}}\right) \leq B\left(d_{\mathcal{S}}(X, Y)+\beta\right)
$$

for some constants $A, B$ and numbers $\alpha$ and $\beta$ which can be made small by refining the samplings. Moreover, it may happen that relation (3) holds with a certain probability. This is exactly the relationship found above for the rigid case and now extended for general isometries.

\subsection{The General Case}

The theory introduced by Gromov addresses the concept of metric approximation between metric spaces. When dealing with discrete metric spaces, as those arising from samplings or coverings of continuous ones, it is convenient to introduce another distance between them which ultimately is the one we compute for point clouds, see $\S 3.6$ ahead. For discrete metric spaces (both of cardinality $n)\left(\mathbb{X}=\left\{x_{1}, \ldots, x_{n}\right\}, d_{\mathbb{X}}\right)$ and $\left(\mathbb{Y}=\left\{y_{1}, \ldots, y_{n}\right\}, d_{\mathbb{Y}}\right)$ we define the distance: ${ }^{4}$

$$
d_{\mathcal{J}}(\mathbb{X}, \mathbb{Y}):=\min _{\pi \in \mathcal{P}_{n}} \max _{1 \leq i, j \leq n} \frac{1}{2}\left|d_{\mathbb{X}}\left(x_{i}, x_{j}\right)-d_{\mathbb{Y}}\left(y_{\pi_{i}}, y_{\pi_{j}}\right)\right|
$$

where $\mathcal{P}_{n}$ stands for the set of all $n \times n$ permutations of $\{1, \ldots, n\}$. A permutation $\pi$ provides the correspondence between the points in the sets, and $\left|d_{\mathbb{X}}\left(x_{i}, x_{j}\right)-d_{\mathbb{Y}}\left(y_{\pi_{i}}, y_{\pi_{j}}\right)\right|$ gives the pointwise distance/disparity once this correspondence has been assumed.

It is evident that one has, by virtue of property 5 from Proposition 1

$$
d_{\mathcal{G H}}(\mathbb{X}, \mathbb{Y}) \leq d_{\mathcal{J}}(\mathbb{X}, \mathbb{Y})
$$

Moreover, we easily derive the following, whose usefulness will be made evident in $\S 3$.

Corollary 2 Let $\left(X, d_{X}\right)$ and $\left(Y, d_{Y}\right)$ be compact metric spaces. Let $\mathbb{X}=\left\{x_{1}, \ldots, x_{n}\right\} \subset X$ and $\mathbb{Y}=\left\{y_{1}, \ldots, y_{n}\right\} \subset Y$, such that $B_{X}\left(\mathbb{X}, R_{X}\right)=X$ and $B_{Y}\left(\mathbb{Y}, R_{Y}\right)=Y$ (the point clouds provide $R_{X}$ and $R_{Y}$ coverings respectively). Then

$$
d_{\mathcal{G} \mathcal{H}}(X, Y) \leq R_{X}+R_{Y}+d_{\mathcal{J}}(\mathbb{X}, \mathbb{Y})
$$

with the understanding that $d_{\mathbb{X}}=\left.d_{X}\right|_{\mathbb{X} \times \mathbb{X}}$ and $d_{\mathbb{Y}}=\left.d_{Y}\right|_{\mathbb{Y} \times \mathbb{Y}}$.

\footnotetext{
${ }^{4}$ One can easily check that this is really a distance.
} 
Remark 2 This result tells us that if we manage to find coverings of $X$ and $Y$ for which the distance $d_{\mathcal{J}}$ is small, then if the radius radii those coverings are also small, the underlying manifolds $X$ and $Y$ sampled by these point clouds must be close in a metric sense. Another way of interpreting this is that we will never see a small value of $d_{\mathcal{J}}(\mathbb{X}, \mathbb{Y})$ whenever $d_{\mathcal{G H}}(X, Y)$ is big, a simple statement with practical value, since we will only be able to look at values of $d_{\mathcal{J}}$, which depend on the point clouds. This is because, in contrast with $d_{\mathcal{G H}}($,$) , the distance d_{\mathcal{J}}$ is (approximately) computable from the point clouds, see $\S 3.6$. We also need to bound $d_{\mathcal{G H}}(X, Y)$ with $d_{\mathcal{J}}$ from below, as done next.

We now introduce some additional notation regarding coverings of metric spaces. Given a metric space $\left(X, d_{X}\right)$, the discrete subset $N_{X, n}^{(R, s)}$ denotes a set of points $\left\{x_{1}, \ldots, x_{n}\right\} \subset X$ such that (1) $B_{X}\left(N_{X, n}^{(R, s)}, R\right)=X$, and $(2) d_{X}\left(x_{i}, x_{j}\right) \geq s$ whenever $i \neq j$. In other words, the set constitutes a R-covering and the points in the set are not too close to each other.

Remark 3 For each $r>0$, denote by $N(r, X)$ the minimum number of closed balls of radii $r$ needed to cover $X$. Then, ([46], Chapter 10), we can actually show that the class $\left(\mathcal{M}, d_{\mathcal{G H}}\right)$ of all compact metric spaces $X$ whose covering number $N(r, X)$ are bounded for all (small) positive $r$ by a function $N:\left(0, C_{1}\right) \rightarrow(0, \infty)$ is totally bounded. This means that given $\rho>0$, there exist a finite positive integer $k(\rho)$ and compact metric spaces $X_{1}, \ldots, X_{k(\rho)} \in \mathcal{M}$ such that for any $X \in \mathcal{M}$ one can find $i \in\{1, \ldots, k(\rho)\}$ such that $d_{\mathcal{G H}}\left(X, X_{i}\right) \leq \rho$. This is very interesting from the point of view of applications since it gives formal justification to classification problem of metric spaces. For example, in a system of storage/retrieval of faces/information manifolds, this concept permits the design of a clustering procedure for the shapes.

The following Proposition will also be fundamental for our computational framework in $\S 3$.

Proposition $3([24])$ Let $\left(X, d_{X}\right)$ and $\left(Y, d_{Y}\right)$ be any pair of given compact metric spaces and let $\eta=d_{\mathcal{G H}}(X, Y)$. Also, let $N_{X, n}^{(R, s)}=\left\{x_{1}, \ldots, x_{n}\right\}$ be given. Then, given $\alpha>0$ there exist points $\left\{y_{1}^{\alpha}, \ldots, y_{n}^{\alpha}\right\} \subset Y$ such that

1. $d_{\mathcal{J}}\left(N_{X, n}^{(R, s)},\left\{y_{1}^{\alpha}, \ldots, y_{n}^{\alpha}\right\}\right) \leq(\eta+\alpha)$

2. $B_{Y}\left(\left\{y_{1}^{\alpha}, \ldots, y_{n}^{\alpha}\right\}, R+2(\eta+\alpha)\right)=Y$

3. $d_{Y}\left(y_{i}^{\alpha}, y_{j}^{\alpha}\right) \geq s-2(\eta+\alpha)$ for $i \neq j$.

Remark 4 This proposition tells us that if the metric spaces happen to be sufficiently close in a metric sense, then given a s-separated covering on one of them, one can find a ( $s^{\prime}$-separated) covering in the other metric space such that $d_{\mathfrak{J}}$ between those coverings (point clouds) is also small. This, in conjunction with Remark 2, proves that in fact our goal of trying to determine the metric similarity of metric spaces based on discrete observations of them is, so far, a (theoretically) well posed problem.

Since by Tychonoff's Theorem the $n$-fold product space $Y \times \ldots \times Y$ is compact, if $s-2 \eta \geq c>0$ for some positive constant $c$, by passing to the limit along the subsequences of $\left\{y_{1}^{\alpha}, \ldots, y_{n}^{\alpha}\right\}_{\{\alpha>0\}}$ (if needed) above one can assume the existence of a set of different points $\left\{\bar{y}_{1}, \ldots, \bar{y}_{n}\right\} \subset Y$ such that $d_{\mathcal{J}}\left(\left\{\bar{y}_{1}, \ldots, \bar{y}_{n}\right\}, N_{X, n}^{(R, s)}\right) \leq \eta, \min _{i \neq j} d_{Y}\left(\bar{y}_{i}, \bar{y}_{j}\right) \geq s-2 \eta>0$, and $B_{Y}\left(\left\{\bar{y}_{1}, \ldots, \bar{y}_{n}\right\}, R+2 \eta\right)=Y$. 
Since we are only given finite sets of points sampled from each metric space, the existence of $\left\{\bar{y}_{1}, \ldots, \bar{y}_{n}\right\}$ guaranteed by Proposition 3 and Remark 4 doesn't seem to make our life a lot easier since those points could very well not be contained in our given finite datasets. The simple idea of using a triangle inequality (with metric $d_{\mathcal{J}}$ ) to deal with this does not work in principle, since one can find, for the same underlying space, two covering nets whose $d_{\mathcal{J}}$ distance is not small, see $[10,38]$. Let us explain this in more detail. Assume that as input we are given two finite sets of points $\mathbb{X}_{m}$ and $\mathbb{Y}_{m}$ on two metric spaces, $X$ and $Y$ respectively, which we assume to be isometric. Then the results above ensure that for any given $N_{X, n}^{(R, s)} \subset \mathbb{X}_{m}$ there exists a $N_{Y, n}^{(R, s)} \subset Y$ such that $d_{\mathcal{J}}\left(N_{X, n}^{(R, s)}, N_{Y, n}^{(R, s)}\right)=0$. However, it is clear that this $N_{Y, n}^{(R, s)}$ has no reason to be contained in the given point cloud $\mathbb{Y}_{m}$. The obvious idea would be try to rely on some kind of property of independence on the sample representing a given metric space, namely that for any two different covering nets $N_{1}$ and $N_{2}$ (of the same cardinality and with small covering radii) of $X$ the distance $d_{\mathcal{J}}\left(N_{1}, N_{2}\right)$ is also small. If this were granted, we could proceed as follows:

$$
\begin{aligned}
d_{\mathcal{J}}\left(N_{X, n}^{(R, s)}, N_{Y, n}^{(\hat{R}, \hat{s})}\right) & \leq d_{\mathcal{J}}\left(N_{X, n}^{(R, s)}, N_{Y, n}^{(R, s)}\right)+d_{\mathcal{J}}\left(N_{Y, n}^{(\hat{R}, \hat{s})}, N_{Y, n}^{(R, s)}\right) \\
& =0+\operatorname{small}(R, \hat{R})
\end{aligned}
$$

where $\operatorname{small}(R, \hat{R})$ is small number depending only on $R$ and $\hat{R}$. The property we fancy to rely upon was conjectured by Gromov in [25] (see also [50]) and disproved by Burago \& Kleiner in [10] and Mc.Mullen in [38], see also [43] for certain positive results. Their counterexamples are for separated covering nets in $\mathbb{Z}^{2}$. It is not known whether one can construct counterexamples for compact metric spaces, or if there exists a characterization of a family of $n$-points separated covering nets of a given compact metric space such that any two of them are at a small $d_{\mathcal{J}}$-distance which can be somehow controlled with $n$. A first step towards this is the density condition introduced in [11].

If counterexamples didn't exist for compact metric spaces, then the above inequality would be sufficient. Without assuming this, we give below an argument which tackles the problem in a probabilistic way. In other words, we use a probabilistic approach to bound $d_{\mathcal{J}}$ for two different samples from a given metric space. For this, we pay the price of assuming the existence of a measure which comes with our metric space. ${ }^{5}$ On the other hand, probabilistic frameworks are natural for (maybe noisy) random samples of manifolds as obtained in real applications. ${ }^{6}$

\subsection{A Probabilistic Setting for Submanifolds of $\mathbb{R}^{d}$}

We now limit ourself to smooth submanifolds of $\mathbb{R}^{d}$, although the work can be extended to more general metric spaces, see further comments in $\S 5$. In what follows, for an event $\mathcal{E}, \mathbf{P}(\mathcal{E})$ will denote its probability and for a random variable $X, \mathbf{E}(X)$ will denote its expected value.

Let $Z$ be a smooth and compact submanifold of $\mathbb{R}^{d}$ with intrinsic (geodesic) distance function $d_{Z}(\cdot, \cdot)$. We can now speak more freely about points $\left\{z_{i}\right\}_{i=1}^{m}$ sampled uniformly from $X$ : We say that the random point $\widehat{z}$ is uniformly distributed on $Z$ if for any measurable $C \subset Z, \mathbf{P}(\widehat{z} \in C)=\frac{\mathbf{a}(C)}{\mathbf{a}(Z)}$, where $\mathbf{a}(B)$ denotes the area of the measurable set $B \subset Z$. This uniform probability measure

\footnotetext{
${ }^{5}$ In the present report we therefore deal only with the case of sub-manifolds of $\mathbb{R}^{d}$.

${ }^{6}$ In more generality, data are acquired by sensors or arrays of sensors which return a value in $\mathbb{R}^{d}$ for some $d \geq 1$. The acquisition process or the sensors themselves might be subject to some perturbations (miscalibrations of mechanical parts of a 3D-scanner, electric noise in electrodes, etc). Under the assumption of existence of an underlying structure from which the data are sampled, it therefore seems sensible to introduce a probability measure which models the acquisition process.
} 
can be replaced by other probability measures which for example adapt to the geometry of the underlying surface, and the framework here developed can be extended to those as well.

Let $\mathbb{Z}=\left\{z_{1}, \ldots, z_{n}\right\}$ and $\mathbb{Z}^{\prime}=\left\{z_{1}^{\prime}, \ldots, z_{n}^{\prime}\right\}$ be two discrete subsets of $Z$ (two point clouds). For any permutation $\pi \in \mathcal{P}_{n}$ and $i, j \in\{1, \ldots, n\}$,

$$
\left|d_{Z}\left(z_{i}, z_{j}\right)-d_{Z}\left(z_{\pi_{i}}^{\prime}, z_{\pi_{j}}^{\prime}\right)\right| \leq d_{Z}\left(z_{i}, z_{\pi_{i}}^{\prime}\right)+d_{Z}\left(z_{j}, z_{\pi_{j}}^{\prime}\right)
$$

and therefore we have

$$
d_{\mathcal{B}}^{Z}\left(\mathbb{Z}, \mathbb{Z}^{\prime}\right):=\min _{\pi \in \mathcal{P}_{n}} \max _{k} d_{Z}\left(z_{k}, z_{\pi_{k}}^{\prime}\right) \geq d_{\mathcal{J}}\left(\mathbb{Z}, \mathbb{Z}^{\prime}\right)
$$

This is known as the Bottleneck Distance between $\mathbb{Z}$ and $\mathbb{Z}^{\prime}$, both being subsets of $Z$. This is one possible way of measuring distance between two different samples of the same metric space. ${ }^{7}$

Instead of dealing with (7) deterministically, after imposing conditions on the underlying metric spaces $X$ and $Y$, we derive probabilistic bounds for the left hand side. We also make evident that by suitable choices of the relations among the different parameters (such as coverage radius and separation), this probability can be chosen at will. This result is then used to bound the distance $d_{\mathcal{J}}$ between two point cloud samples of a given metric space, thereby leading to the type of bound expressed in Equation (7) and from this, the bounds on the original Gromov-Hausdorff distance between the underlying objects.

We introduce the Voronoi diagram $\mathcal{V}(\mathbb{Z})$ on $Z$, determined by the points in $\mathbb{Z}$ (see for example [34]). The $i$-th Voronoi cell of the Voronoi diagram defined by $\left\{z_{1}, \ldots, z_{n}\right\} \subset Z$ is given by

$$
A_{i}:=\left\{z \in Z \mid d_{Z}\left(z_{i}, z\right)<\min _{j \neq i} d_{Z}\left(z_{j}, z\right)\right\}
$$

We then have $Z=\bigsqcup_{k=1}^{n} \overline{A_{k}}$.

Lemma 1 1. If the points $\left\{z_{1}, \ldots, z_{n}\right\}$ are s-separated, then for any $1 \leq i \leq n, B_{Z}\left(z_{i}, \frac{s}{2}\right) \subset A_{i}$.

2. If the points $\left\{z_{1}, \ldots, z_{n}\right\}$ constitute a $R$-covering of $Z$, then $A_{i} \subseteq B_{Z}\left(z_{i}, R\right)$ for all $i=1, \ldots, n$.

\section{Proof:}

To prove 1 . first note that for any $z \in Z$ and $i \neq j, d_{Z}\left(z, z_{i}\right)+d_{Z}\left(z, z_{j}\right) \geq s$ by the triangle inequality. Assume in particular that $z \in B_{Z}\left(z_{i}, \frac{s}{2}\right)$, then $d_{Z}\left(z, z_{i}\right)<\frac{s}{2}$ and $d_{Z}\left(z, z_{j}\right)>\frac{s}{2}$ for all $j \neq i$, then $z \in A_{i}$. To prove 2. assume $z \in A_{i}$ but $z \notin B_{Z}\left(z_{i}, R\right)$, that is $d_{Z}\left(z, z_{i}\right) \geq R$. But since $\left\{z_{1}, \ldots, z_{n}\right\}$ is a $R$-covering of $Z, z$ must belong to a certain $B_{Z}\left(x_{k}, R\right)$ for some $k \neq i$, that is $d_{Z}\left(z, z_{k}\right)<R$. But then $z$ is closer to $z_{k}$ than to $z_{i}$, which contradicts $z \in A_{i}$.

We consider $\mathbb{Z}$ to be fixed, and we assume $\mathbb{Z}^{\prime}=\left\{z_{1}^{\prime}, \ldots, z_{n}^{\prime}\right\}$ to be chosen from a set $\mathbb{Z}_{m} \subset Z$ consisting of $m \gg n$ i.i.d. points sampled uniformly from $Z$.

We first want to find, amongst points in $\mathbb{Z}_{m}, n$ different points $\left\{z_{i_{1}}, \ldots, z_{i_{n}}\right\}$ such that each of them belongs to one Voronoi cell, $\left\{z_{i_{k}} \in A_{k}\right.$ for $\left.k=1, \ldots, n\right\}$. We provide lower bounds for $\mathbf{P}\left(\#\left(A_{k} \cap \mathbb{Z}_{m}\right) \geq 1, \quad 1 \leq k \leq n\right)$, the probability of this happening.

We can see the event as if we collected points inside all the Voronoi cells, a case of the Coupon Collecting Problem, see [19]. We buy merchandise at a coupons-giving store until we have collected all possible types of coupons. The next Lemma presents the basic results we need about this concept. These results are due to Von Schilling ([51]) and Borwein and Hijab ([4]).

\footnotetext{
${ }^{7}$ In [43], this distance is used to establish the equivalence (according to this notion) of separated nets in certain Hyperbolic metric spaces.
} 
Lemma 2 (Coupon Collecting) If there are $n$ different coupons one wishes to collect, such that the probability of seeing the $k$-th coupon is $p_{k} \in(0,1)$, (let $\vec{p}=\left(p_{1}, \ldots, p_{n}\right)$ ), and one obtains samples of all of them in an independent way then:

1. ([51]) The probability $P_{\vec{p}}(n, m)$ of having collected all $n$ coupons after $m$ trials is given by

$$
P_{\vec{p}}(n, m)=1-S_{n}\left(\sum_{j=2}^{n}(-1)^{j}\left(\sum_{k=j}^{n} p_{k}\right)^{m}\right)
$$

where the symbol $S_{n}$ means that we consider all possible combinations of the $n$ indices in the expression being evaluated. ${ }^{8}$

2. ([4]) The expected value of the number of trials needed to collect all the coupons is given by

$$
E_{\vec{p}}(n)=\mathbf{E}\left(\max _{1 \leq i \leq n} \frac{X_{i}}{p_{i}}\right)
$$

where $X_{i}$ are independent positive random variables satisfying $\mathbf{P}\left(X_{i}>t\right)=e^{-t}$ for $t \geq 0$ and $1 \leq i \leq n$.

For $n \in \mathbb{N}$ let $h_{n}:=\sum_{i=1}^{n} i^{-1}$.

Corollary $3 \quad P_{\vec{p}}(n, m) \geq 1-\frac{h_{n}}{m \cdot \min _{k} p_{k}}$.

\section{Proof:}

By Markov's inequality, $1-P_{\vec{p}}(n, m) \leq \frac{E_{\vec{p}(n)}}{m}$. Now, note that $E_{\vec{p}}(n)$ is decreasing in each $p_{k}$ for $p_{k} \geq 0$, then it is clear that $E_{\vec{p}}(n) \leq \frac{E_{\vec{l}}(n)}{\min _{k} p_{k}}$. On the other hand, it is easy to compute by direct probabilistic calculation that $E_{\frac{\overrightarrow{1}}{n}}(n)=n h_{n}$. We conclude by noting that, by $(11), c E_{c \vec{p}}(n)=E_{\vec{p}}(n)$ for any $c>0$.

We now directly use these results to bound the bottleneck distance.

Theorem 1 Let $\left(Z, d_{Z}\right)$ be a smooth compact submanifold of $\mathbb{R}^{d}$. Given a covering $N_{Z, n}^{(R, s)}$ of $Z$ with separation $s>0$ and a number $p \in(0,1)$, there exists a positive integer $m=m_{n}(p)$ such that if $\mathbb{Z}_{m}=\left\{z_{k}\right\}_{k=1}^{m}$ is a sequence of $i . i . d$. points sampled uniformly from $Z$, with probability $p$ one can find a set of $n$ different indices $\left\{i_{1}, \ldots, i_{n}\right\} \subset\{1, \ldots, m\}$ with

$$
\begin{aligned}
& \qquad d_{\mathcal{B}}^{Z}\left(N_{Z, n}^{(R, s)},\left\{z_{i_{1}}, \ldots, z_{i_{n}}\right\}\right) \leq R \quad \text { and } \quad Z=\bigcup_{k=1}^{n} B_{Z}\left(z_{i_{k}}, 2 R\right) . \\
& \text { Moreover, } m_{n}(p) \leq\left[\frac{h_{n}}{\min _{z} \mathbf{a}\left(B_{Z}\left(z, \frac{s}{2}\right)\right)} \frac{\mathbf{a}(Z)}{1-p}\right]+1 .{ }^{9}
\end{aligned}
$$

This result can also be seen the other way around: For a given $m$, the probability of finding the aforementioned subset in $\mathbb{Z}_{m}$ is $P_{\vec{p}_{Z}}(n, m)$ as given by $(10)$, for suitably defined $\vec{p}_{Z}$. The precise form of $\vec{p}_{Z}$ can be understood from the proof.

\section{Proof:}

Let $N_{Z, n}^{(R, s)}=\left\{\widehat{z}_{1}, \ldots, \widehat{z}_{n}\right\}$. We consider the coupon collecting problem in which the $k$-th coupon has been

${ }^{8}$ For example $S_{3}\left(\left(p_{1}+p_{2}\right)^{k}\right)=\left(p_{1}+p_{2}\right)^{k}+\left(p_{1}+p_{3}\right)^{k}+\left(p_{2}+p_{3}\right)^{k}$.

${ }^{9}$ For real $x,[x]$ stands for the largest integer not greater that $x$. 
acquired at least once if $\#\left\{\mathbb{Z}_{m} \cap A_{k}\right\} \geq 1$, where $A_{k}$ is the $k$-th cell of the Voronoi partition corresponding to the covering net $N_{Z, n}^{(R, s)}$. The components of the probability vector $\vec{p}$ are given by $p_{k}=\frac{\mathrm{a}\left(A_{k}\right)}{\mathrm{a}(Z)}$ for $k=1, \ldots, n$. Using the fact that (10) is increasing in the number of trials $m,{ }^{10}$ we see that given $p$ we can find a positive integer $M$ such that for $m \geq M$

$$
\mathbf{P}\left(\bigcap_{k=1}^{n}\left\{\#\left\{\mathbb{Z}_{m} \cap A_{k}\right\} \geq 1\right\}\right) \geq p
$$

Discarding points when more than one has been found inside the same $A_{k}$, we can obtain with probability at least $p$, exactly one point inside each $A_{k}$. Let $i_{1}, \ldots, i_{n}$ be indices such that $z_{i_{k}} \in A_{k}$ for $k=1, \ldots, n$. Then $d_{\mathcal{B}}^{Z}\left(\left\{z_{i_{1}}, \ldots, z_{i_{n}}\right\}, N_{Z, n}^{(R, s)}\right) \leq \max _{z \in \bar{A}_{k}} d_{Z}\left(z, \widehat{z}_{k}\right)$, since by Lemma $1, A_{k} \subseteq B_{Z}\left(\widehat{z}_{k}, R\right)$, and this concludes the proof of the first claim. Also, by the very same steps plus the triangle inequality we prove that $\left\{z_{i_{1}}, \ldots, z_{i_{n}}\right\}$ constitutes a $2 R$-covering of $Z$. Finally, note that by Corollary $3, P_{\vec{p}}(n, m) \geq p$ for $m \geq \frac{h_{n}}{(1-p) \min _{k} p_{k}}$. Since again by Lemma $1, \mathbf{a}\left(A_{k}\right) \geq \min _{z \in Z} \mathbf{a}\left(B_{Z}\left(z, \frac{s}{2}\right)\right)$, the last claim follows.

Corollary 4 Let $X$ and $Y$ compact submanifolds of $\mathbb{R}^{d}$. Let $N_{X, n}^{(R, s)}$ be a covering of $X$ with separation s such that for some positive constant $c, s-2 d_{\mathcal{G H}}(X, Y)>c$. Then, given any number $p \in(0,1)$, there exists a positive integer $m=m_{n}(p)$ such that if $\mathbb{Y}_{m}=\left\{y_{k}\right\}_{k=1}^{m}$ is a sequence of i.i.d. points sampled uniformly from $Y$, we can find, with probability at least $p$, a set of $n$ different indices $\left\{i_{1}, \ldots, i_{n}\right\} \subset\{1, \ldots, m\}$ such that

$$
\begin{aligned}
& d_{\mathcal{J}}\left(N_{X, n}^{(R, s)},\left\{y_{i_{1}}, \ldots, y_{i_{n}}\right\}\right) \leq 3 d_{\mathcal{G H}}(X, Y)+R \quad \text { and } \quad Y=\bigcup_{k=1}^{n} B_{Y}\left(y_{i_{k}}, 2\left(R+2 d_{\mathcal{G}}(X, Y)\right)\right) . \\
& \text { Moreover, } m_{n}(p) \leq\left[\frac{h_{n}}{\min _{y} \mathbf{a}\left(B_{Y}\left(y, \frac{c}{2}\right)\right)} \frac{\mathbf{a}(Y)}{1-p}\right]+1 .
\end{aligned}
$$

\section{Proof:}

Let $\eta=d_{\mathcal{G} \mathcal{H}}(X, Y)$. Following Remark 4 , we can find a $(R+2 \eta, s-2 \eta) n$-covering of $Y$, which we denote by $N_{Y, n}^{(\tilde{R}, \tilde{s})}$, such that $d_{\mathcal{J}}\left(N_{X, n}^{(R, s)}, N_{Y, n}^{(\tilde{R}, \tilde{s})}\right) \leq \eta$. Let, as in Theorem $1, m=m_{n}(p)$ be such that for any i.i.d. set of points $\mathbb{Y}_{m}=\left\{y_{1}, \ldots, y_{m}\right\}$ uniformly sampled from $Y$ one has

$$
\mathbf{P}\left(\exists\left\{y_{i_{1}}, \ldots, y_{i_{n}}\right\} \subset \mathbb{Y}_{m}: d_{\mathcal{B}}^{Y}\left(N_{Y, n}^{(\tilde{R}, \tilde{s})},\left\{y_{i_{1}}, \ldots, y_{i_{n}}\right\}\right) \leq \tilde{R}\right) \geq p
$$

where $i_{1}, \ldots, i_{n}$ are different indices. Let $N_{Y, n} \subset Y$ be a set of $n$ different points. Then, using the triangle inequality

$$
\begin{aligned}
d_{\mathcal{J}}\left(N_{X, n}^{(R, s)}, N_{Y, n}\right) & \leq d_{\mathcal{J}}\left(N_{X, n}^{(R, s)}, N_{Y, n}^{(\tilde{R}, \tilde{s})}\right)+d_{\mathcal{J}}\left(N_{Y, n}, N_{Y, n}^{(\tilde{R}, \tilde{s})}\right) \\
& \leq \eta+d_{\mathcal{B}}^{Y}\left(N_{Y, n}, N_{Y, n}^{(\tilde{R}, \tilde{s})}\right)
\end{aligned}
$$

Hence we obtain, by Theorem 1 ,

$$
\mathbf{P}\left(\exists N_{Y, n} \subset \mathbb{Y}_{m}: d_{\mathcal{J}}\left(N_{X, n}^{(\tilde{R}, \tilde{s})}, N_{Y, n}\right) \leq \eta+\tilde{R}\right) \geq p .
$$

The other claims follow just like in the proof of Theorem 1.

\footnotetext{
${ }^{10}$ Something obvious for which in, principle, we do not need to know the exact expression (10).
} 
Remark 5 The probability $P_{\vec{p}_{Y}}(n, m)$ itself (or $m_{n}(p)$ ) depends on $d_{\mathcal{G H}}(X, Y)$ through the constant $c$, see an example of the application of this ideas in $§ 3.2$ ahead.

One can write down the following useful bound

$$
P_{\vec{p}_{Y}}(n, m) \geq 1-\frac{h_{n}}{m \cdot \min _{y \in Y} B_{Y}\left(y, \frac{c}{2}\right)} \mathbf{a}(Y)
$$

which was implicitly used in the proof of Theorem 1. It is sensible to assume one is interested in performing the recognition/classification task for a number of objects which satisfy certain conditions, that is, tune the framework to a particular class of objects. In particular, suppose the class is characterized, among other conditions, by an upper bound on the sectional curvatures. For small $r>0$ this allows, via Bishop-Günther's Theorem, to obtain a lower bound on $\min _{z} \mathbf{a}\left(B_{Z}(z, r)\right)$ valid for all objects $Z$ in the class. This in turn can be used to calibrate the system to provide any pre-specified probability $p$ as in Corollary 4 for any two objects within the class, see §3.2 for a more detailed presentation of these ideas via an example.

A rougher estimate of the value of $m_{n}(p)$ alluded to in Corollary 4 can be obtained using the value of $E_{\vec{p}}(n)$ when all the coupons are equally likely: $m \simeq E_{\frac{\vec{l}}{n}}(n)=n \cdot h_{n} \simeq n \ln n$.

This concludes the main theoretical foundation of our proposed framework. Now, we must devise a computational procedure which allows us to actually find the subset $N_{Y, n}$ inside the given point cloud $\mathbb{Y}_{m}$ when it exists, or at least find it with a large probability. Note that in practise we can only access metric information, that is, interpoint distances. A stronger result in the same spirit of Theorem 1 should take into account possible self-isometries of $X(Y)$, which would increase the probability of finding a net which achieves small $d_{\mathcal{J}}$ distance to the fixed one. We present such a computational framework next.

\section{Computational Foundations}

There are a number of issues that must be dealt with in order to develop an algorithmic procedure from the theoretical results previously presented. These are now addressed.

\subsection{Initial Considerations}

In practise our input consists of two statistically independent point clouds $\mathbb{X}_{m}$ and $\mathbb{Y}_{m^{\prime}}$ each of them composed of i.i.d. points sampled uniformly from $X$ and $Y$, respectively. For a positive integer $n \ll \min \left(m, m^{\prime}\right)$ we construct good coverings $N_{X, n}^{(R, s)}$ of $X$ and $N_{Y, n}^{\left(R^{\prime}, s^{\prime}\right)}$ of $Y$, respectively. Actually, $R, s, R^{\prime}$ and $s^{\prime}$ all depend on $n$, and we should choose $n$ such that $R$ and $R^{\prime}$ are small enough to make our bounds useful, see the additional computations below. Details on how we construct these coverings are provided in Section $§ 3.4$. We will assume, without loss of generality, that this coverings are statistically independent of $\mathbb{X}_{m}$ and $\mathbb{Y}_{m^{\prime}}$.

It is convenient to introduce the following additional notation: For a set of points $\mathbb{Z}_{q}=\left\{z_{k}\right\}_{k=1}^{q}$ and for a set of indices $I_{u}=\left\{i_{1}, \ldots, i_{u}\right\} \subset\{1, \ldots, q\}$, let $\mathbb{Z}_{q}\left[I_{u}\right]$ denote the subset $\left\{z_{i_{1}}, \ldots, z_{i_{u}}\right\}$ of $\mathbb{Z}_{q}$.

Corollary 4 suggests that in practise we compute the following symmetric expression

$$
d_{\mathcal{F}}\left(\mathbb{X}_{m}, \mathbb{Y}_{m^{\prime}}\right):=\max \left(\min _{J_{n} \subset\{1, \ldots, m\}} d_{\mathcal{J}}\left(N_{X, n}^{(R, s)}, \mathbb{Y}_{m^{\prime}}\left[J_{n}\right]\right), \min _{I_{n} \subset\{1, \ldots, m\}} d_{\mathcal{J}}\left(N_{Y, n}^{\left(R^{\prime}, s^{\prime}\right)}, \mathbb{X}_{m}\left[I_{n}\right]\right)\right)
$$


which depends not only on $\mathbb{X}_{m}$ and $\mathbb{Y}_{m^{\prime}}$ but also on pre-specified covering nets $N_{X, n}^{(R, s)}$ and $N_{Y, n}^{\left(R^{\prime}, s^{\prime}\right)}$. However we prefer to omit this dependence in the list of arguments in order to keep the notation simpler.

Then, $d_{\mathcal{F}}\left(\mathbb{X}_{m}, \mathbb{Y}_{m^{\prime}}\right)$ upper bounds $d_{\mathcal{G H}}\left(\mathbb{X}_{m}, \mathbb{Y}_{m^{\prime}}\right)$, something we need to require. In fact, for any $I_{n} \subset\{1, \ldots, m\}$

$$
\begin{aligned}
d_{\mathcal{G H}}\left(\mathbb{X}_{m}, \mathbb{Y}_{m^{\prime}}\right) & \leq d_{\mathcal{G H}}\left(\mathbb{X}_{m}, \mathbb{X}_{m}\left[I_{n}\right]\right)+d_{\mathcal{G H}}\left(\mathbb{X}_{m}\left[I_{n}\right], \mathbb{Y}_{m^{\prime}}\right) \\
& \leq d_{\mathcal{G H}}\left(\mathbb{X}_{m}, \mathbb{X}_{m}\left[I_{n}\right]\right)+d_{\mathcal{G H}}\left(\mathbb{X}_{m}\left[I_{n}\right], N_{Y, n}^{\left(R^{\prime}, s^{\prime}\right)}\right)+d_{\mathcal{G H}}\left(N_{Y, n}^{\left(R^{\prime}, s^{\prime}\right)}, \mathbb{Y}_{m^{\prime}}\right) \\
& \leq d_{\mathcal{H}}^{X}\left(\mathbb{X}_{m}, \mathbb{X}_{m}\left[I_{n}\right]\right)+d_{\mathcal{J}}\left(\mathbb{X}_{m}\left[I_{n}\right], N_{Y, n}^{\left(R^{\prime}, s^{\prime}\right)}\right)+R^{\prime}
\end{aligned}
$$

Now, considering $I_{n}$ such that $d_{\mathcal{J}}\left(\mathbb{X}_{m}\left[I_{n}\right], N_{Y, n}^{\left(R^{\prime}, s^{\prime}\right)}\right)=\min _{I_{n} \subset\{1, \ldots, m\}} d_{\mathcal{J}}\left(N_{Y, n}^{(R, s)}, \mathbb{X}_{m}\left[I_{n}\right]\right)$, we find

$$
d_{\mathcal{G H}}\left(\mathbb{X}_{m}, \mathbb{Y}_{m^{\prime}}\right) \leq d_{\mathcal{H}}^{X}\left(\mathbb{X}_{m}, \mathbb{X}_{m}\left[I_{n}\right]\right)+d_{\mathcal{F}}\left(\mathbb{X}_{m}, \mathbb{Y}_{m^{\prime}}\right)+R^{\prime}
$$

Symmetrically, we also obtain for $J_{n}$ such that $d_{\mathcal{J}}\left(\mathbb{Y}_{m}\left[J_{n}\right], N_{X, n}^{(R, s)}\right)=\min _{J_{n} \subset\left\{1, \ldots, m^{\prime}\right\}} d_{\mathcal{J}}\left(N_{X, n}^{(R, s)}, \mathbb{Y}_{m^{\prime}}\left[J_{n}\right]\right)$

$$
d_{\mathcal{G H}}\left(\mathbb{X}_{m}, \mathbb{Y}_{m^{\prime}}\right) \leq d_{\mathcal{H}}^{Y}\left(\mathbb{Y}_{m^{\prime}}, \mathbb{Y}_{m^{\prime}}\left[J_{n}\right]\right)+d_{\mathcal{F}}\left(\mathbb{X}_{m}, \mathbb{Y}_{m^{\prime}}\right)+R
$$

Hence, combining the last two expressions

$$
\begin{aligned}
d_{\mathcal{G H}}\left(\mathbb{X}_{m}, \mathbb{Y}_{m^{\prime}}\right) & \leq d_{\mathcal{F}}\left(\mathbb{X}_{m}, \mathbb{Y}_{m^{\prime}}\right) \\
& +\min \left(d_{\mathcal{H}}^{X}\left(\mathbb{X}_{m}, \mathbb{X}_{m}\left[I_{n}\right]\right), d_{\mathcal{H}}^{Y}\left(\mathbb{Y}_{m^{\prime}}, \mathbb{Y}_{m^{\prime}}\left[J_{n}\right]\right)\right) \\
& +\max \left(R, R^{\prime}\right)
\end{aligned}
$$

what implies (Corollary 1) a similar upper bound for $d_{\mathcal{G H}}(X, Y)$. In fact, let $r_{m}:=d_{\mathcal{H}}^{X}\left(X, \mathbb{X}_{m}\right)$ and $r_{m^{\prime}}:=d_{\mathcal{H}}^{Y}\left(Y, \mathbb{Y}_{m^{\prime}}\right)$, then

$$
\begin{aligned}
d_{\mathcal{G H}}(X, Y) & \leq d_{\mathcal{F}}\left(\mathbb{X}_{m}, \mathbb{Y}_{m^{\prime}}\right) \\
& +\min \left(d_{\mathcal{H}}^{X}\left(\mathbb{X}_{m}, \mathbb{X}_{m}\left[I_{n}\right]\right), d_{\mathcal{H}}^{Y}\left(\mathbb{Y}_{m^{\prime}}, \mathbb{Y}_{m^{\prime}}\left[J_{n}\right]\right)\right) \\
& +\max \left(R, R^{\prime}\right)+r_{m}+r_{m^{\prime}}
\end{aligned}
$$

Let $\Delta_{X}:=d_{\mathcal{H}}^{X}\left(\mathbb{X}_{m}, \mathbb{X}_{m}\left[I_{n}\right]\right)$ and $\Delta_{Y}:=d_{\mathcal{H}}^{Y}\left(\mathbb{Y}_{m^{\prime}}, \mathbb{Y}_{m^{\prime}}\left[J_{n}\right]\right)$. We now deal with the opposite kind of inequality. By Corollary 4 we know that with probability at least $P_{\vec{p}_{X}}(n, m) \times P_{\vec{p}_{Y}}\left(n, m^{\prime}\right)$ we will have both: ${ }^{11}$

$$
d_{\mathcal{F}}\left(\mathbb{X}_{m}, \mathbb{Y}_{m^{\prime}}\right) \leq 3 d_{\mathcal{G H}}(X, Y)+\max \left(R, R^{\prime}\right)
$$

and

$$
\Delta_{X} \leq R^{\prime}+2 d_{\mathcal{G H}}(X, Y) \quad \text { and } \quad \Delta_{Y} \leq R+2 d_{\mathcal{G H}}(X, Y)
$$

and from this it follows in particular that $\min \left(\Delta_{X}, \Delta_{Y}\right) \leq \max \left(R, R^{\prime}\right)+2 d_{\mathcal{G H}}(X, Y)$ with the same probability.

Summing up, we have thus obtained:

\footnotetext{
${ }^{11}$ Because we assumed $\mathbb{X}_{m}$ to be independent from $\mathbb{Y}_{m^{\prime}}$.
} 


$$
d_{\mathcal{G H}}(X, Y)-\alpha\left(R, R^{\prime}, m, m^{\prime}\right) \leq \mathcal{L}\left(\mathbb{X}_{m}, \mathbb{Y}_{m^{\prime}}\right) \stackrel{\text { prob }}{\leq} 5\left(d_{\mathcal{G H}}(X, Y)+\beta\left(R, R^{\prime}\right)\right)
$$

where the symbol $\stackrel{\text { prob }}{\leq}$ means that the inequality holds with probability $P_{\vec{p}_{X}}(n, m) \times P_{\vec{p}_{Y}}\left(n, m^{\prime}\right)$, $\alpha\left(R, R^{\prime}, m, m^{\prime}\right):=\max \left(R, R^{\prime}\right)+\left(r_{m}+r_{m^{\prime}}\right), \beta\left(R, R^{\prime}\right):=\frac{3}{5} \max \left(R, R^{\prime}\right)$ and

$$
\mathcal{L}\left(\mathbb{X}_{m}, \mathbb{Y}_{m^{\prime}}\right):=d_{\mathcal{F}}\left(\mathbb{X}_{m}, \mathbb{Y}_{m^{\prime}}\right)+\min \left(\Delta_{X}, \Delta_{Y}\right)
$$

Remark 6 Note that $\alpha, \beta$ and the probability can be controlled by suitably choosing all the underlying parameters. We have therefore obtained an expression like the one anticipated in Section §2.1, equation (3). The main difference is that we have yet not proved that $\mathcal{L}\left(\mathbb{X}_{m}, \mathbb{Y}_{m^{\prime}}\right)$ can be computed exactly or approximately in practise. In $\S 3.6$ we present a simple algorithm for approximately computing this quantity. We do not provide bounds on the fidelity of the algorithm in this paper. Results in this direction are subject of current efforts.

Remark 7 By a modification of the ideas here presented it may be possible to provide a framework for the recognition of partial objects: One might want to check whether one object is a part of another one. Clearly, in that case, one shouldn't but compute one half of $d_{\mathcal{F}}$. The covering net $N_{Y, n}^{\left(R^{\prime}, s^{\prime}\right)}$ should represent the object which we want to find inside the one represented by $\mathbb{X}_{m}$.

Next, we present a simplified example of the basic ideas discussed so far.

\subsection{An Idealized Example}

Assume that we are trying to detect, amongst a finite number of objects $\left\{X^{i}\right\}_{i=1}^{L}$ belonging to a certain family $\mathcal{A}$, when two objects are isometric. We will assume for simplicity of exposition that we have only two possible cases or hypotheses: (H1) $d_{\mathcal{G H}}\left(X^{i}, X^{j}\right)=0$, and $(\mathrm{H} 2) d_{\mathcal{G H}}\left(X^{i}, X^{j}\right) \geq D$ for some $D>0$ for all $1 \leq i, j \leq L$.

We characterize the family $\mathcal{A}$ as those smooth compact surfaces of $\mathbb{R}^{3}$ such that their Gaussian curvature is bounded from above by some positive constant $K$, and whose total area is bounded from above by some finite constant $A$. Then, for any sufficiently small $c>0$,

$$
\min _{x \in \mathcal{S}} \mathbf{a}\left(B_{\mathcal{S}}(x, c)\right) \geq \frac{2 \pi}{K}(1-\cos (c \sqrt{K}))=: f_{K}(c)
$$

for all $\mathcal{S} \in \mathcal{A}$, by the Bishop-Günther Theorem, see [23]. Note in particular that $f_{K}(c)>0$ for $0<c<\frac{\pi}{\sqrt{K}}$.

For $1 \leq i \leq L$ we will denote by $\mathbb{X}_{m_{i}}^{i}$ the point cloud corresponding to the object $X^{i}$ and $r_{i}$ will denote numbers such that $X \subset B\left(\mathbb{X}_{m_{i}}^{i}, r_{i}\right) .{ }^{12}$

Let $X^{i}$ and $X^{j}$ be any two such objects. We will decide, in this example, that $X^{i}$ and $X^{j}$ are isometric whenever $\mathcal{L}\left(\mathbb{X}_{m_{i}}^{i}, \mathbb{X}_{m_{j}}^{j}\right)$ is smaller than a certain threshold, see equation (17).

Let us fix $\varepsilon>0$. For all $X^{i}$, we choose coverings $N_{X^{i}, n_{i}}^{\left(R_{i}, s_{i}\right)}$ such that $\max _{1 \leq i \leq L} R_{i} \leq \varepsilon$, then $n_{i}$ will be fixed by the procedure one uses to construct those coverings, see $\S 3.4$ ahead. Let $n:=\max _{i} n_{i}$ and $R:=\min _{i} R_{i} \leq \varepsilon$. By adding new points to each of the covering if necessary, we construct new coverings all with $n$ points, covering radius $\varepsilon$ and resulting separation $s_{i}$. Let $s:=\min _{i} s_{i}$.

\footnotetext{
${ }^{12}$ In this example we will neglect the fact that this covering relation will hold with a certain probability.
} 
We are now going to estimate the number of sample points (cardinality of the point clouds) needed for each (all) of the objects in order to be able to detect (H1) with high probability.

According to (17), for any $1 \leq i, j \leq L$ we know that:

- Under (H1), with a probability $Q_{i j}:=P_{\vec{p}_{X i}}\left(n, m_{i}\right) \times P_{\vec{p}_{X j}}\left(n, m_{j}\right)$ we have

$$
\mathcal{L}\left(\mathbb{X}_{m_{i}}, \mathbb{X}_{m_{j}}\right) \leq 3 \varepsilon
$$

- Also, under (H2), assuming $\varepsilon \geq r_{k}$ for $1 \leq k \leq L,{ }^{13}$

$$
\mathcal{L}\left(\mathbb{X}_{m_{i}}, \mathbb{X}_{m_{j}}\right) \geq D-3 \varepsilon
$$

This tells us how to design $\varepsilon$ in relation to $D$ in order to be able to tell both hypotheses apart by computing $\mathcal{L}\left(\mathbb{X}_{m_{i}}, \mathbb{X}_{m_{j}}\right)$. Thus, let $\varepsilon \ll \frac{D}{6} \cdot{ }^{14}$

Now, one wants to impose $Q_{i j}$ to be high, that is $Q_{i j} \geq(1-q)^{2}$ for some small pre-specified $q$. Then, using the comments in Remark 5 , we see that we can for example fix $c:=s$ and estimate the required number of samples for each $X_{i}$ as $m_{i} \geq \frac{h_{n} A}{q \cdot f_{K}\left(\frac{s}{2}\right)}$. In conclusion, one can require that all the point clouds consist of at least $\frac{h_{n} A}{q \cdot f_{K}\left(\frac{s}{2}\right)}$ points (uniformly sampled) from each of the objects.

\subsection{Working with Point Clouds}

All we have are finite sets of points (point clouds) sampled from each metric space, and all our computations must be based on these observations only. Since we made the assumption of randomness in the sampling (and it also makes sense in general to make a random model of the problem, given that the shapes are acquired by a scanner for example), we must relate the number of acquired data points to the coverage properties we wish to have. In other words, and following our theory above, we would like to say that given a desired probability $p_{c}$ and a radius $r_{c}$, there exists a finite $m$ such that the probability of covering all the metric space with $m$ balls (intrinsic or not) of radius $r_{c}$ centered at those $m$ random points is at least $p_{c}$. This kind of characterizations are easy to deal with in the case of submanifolds of $\mathbb{R}^{d}$, where the tuning comes from the curvature bounds available. For this we follow [39]. Let $Z$ be a smooth and compact submanifold of $\mathbb{R}^{d}$ of dimension $k$. Let $\mathbb{Z}_{m} \subset Z$ consist of $m$ i.i.d. points uniformly sampled from $Z$. Let $K$ be an upper bound for the sectional curvatures of $Z$. Then we can prove that for a sequence $r_{m} \rightarrow 0$ such that $r_{m} \gtrsim \frac{\ln m}{m}$ for large $m$

$$
\mathbf{P}\left(d_{\mathcal{H}}^{\mathbb{R}^{d}}\left(Z, \mathbb{Z}_{m}\right)>r_{m}\right) \simeq \frac{1}{\ln m}
$$

Then, since we can also prove, [39], that for any $z \in Z, \delta>0$ small, $B(z, \delta) \cap Z \subset B_{Z}\left(z, C_{K} \delta\right)$, for some constant $C_{K}>1$ depending only on metric properties of $Z$ (curvatures and diameter), we also find

$$
\mathbf{P}\left(d_{\mathcal{H}}^{Z}\left(Z, \mathbb{Z}_{m}\right)>r_{m}\right) \simeq \frac{1}{\ln m}
$$

This relation gives us some guidance about the number points we must sample in order to have a certain covering radius, or to estimate the covering radius in terms of $m$. More precise estimates can be found in the reference mentioned above. An important point to remark is that this kind of relations should hold for the family of shapes we want to work with (in a way similar

\footnotetext{
${ }^{13}$ This is reasonable since $\mathbb{X}_{m_{i}}^{i}$ are supposed to be finer samplings than $N_{X^{i}, n}^{\left(R_{i}, s_{i}\right)}$.

${ }^{14}$ What means that all $R_{i} \ll \frac{D}{6}$.
} 
to the one exposed in $§ 3.2$ ). Therefore, once given bounds on the curvatures and diameters which characterize the family, one can determine a precise probabilistic covering relation for it. We leave the exploitation of this idea for future work.

Given the natural number $n \leq m$ (or eventually $s>0$ ), we use the oracle described in $\S 3.4$ below to find $n$-points from $\mathbb{Z}_{m}$ which constitute a covering of $\mathbb{Z}_{m}$ of the given cardinality $n$ (or of the given separation $s$ ) and of a resulting radius $R$. We denote this set by $N_{\mathbb{Z}_{m}, n}^{(R, s)} \subseteq \mathbb{Z}_{m}$.

\subsection{Finding Coverings}

In order to find the coverings $N_{Z, n}^{(R, s)}$, we use the well known Farthest Point Sampling (FPS) strategy, which we describe next. Suppose we have a dense sampling $\mathbb{Z}_{m}$ of the smooth and compact submanifold $\left(Z, d_{Z}\right)$ of $\mathbb{R}^{d}$ as interpreted by the discussion above. We want to simplify our sampling and obtain a well separated covering net of the space. We also want to estimate the covering radius and separation of our covering net. It is important to obtain subsets which retain as best as possible the metric information contained in the initial point cloud in order to make computational tasks more treatable without sacrificing precision.

We first show a procedure to sample the whole of $Z$. Fix $n$ the number of points we want to have in our simplified point cloud $\mathcal{P}_{n}$. We build $\mathcal{P}_{n}$ recursively. Given $\mathcal{P}_{n-1}$, we select $p \in Z$ such that $d_{Z}\left(p, \mathcal{P}_{n}\right)=\max _{z \in Z} d_{Z}\left(z, \mathcal{P}_{n-1}\right)$ (here we consider of course, geodesic distances). There might exist more than one point which achieves the maximum, we either consider all of them or randomly select one and add it to $\mathcal{P}_{n-1}$. This subsampling procedure has been studied and efficiently implemented in [41] for the case of surfaces represented as point clouds. The FPS procedure satisfies several useful properties as described below.

Let $M\left(\mathcal{P}_{n-1}\right) \subset Z$ denote the set of points $z$ for which $d_{Z}\left(\mathcal{P}_{n-1}, z\right)$ is maximal. We denote by $s_{n}$ and $R_{n}$ the separation and covering radius of $\mathcal{P}_{n} \subset Z$, respectively.

Lemma 3 Let $\mathcal{P}_{n}$ be the set obtained for each $n \geq n_{0}$ according to the $\boldsymbol{F P S}$ strategy starting from $\mathcal{P}_{n_{0}}$, and let $p_{n+1}$ denote any point in $M\left(\mathcal{P}_{n}\right)$. Then, for $n \geq n_{0}$

1. $d_{\mathcal{H}}^{Z}\left(Z, \mathcal{P}_{n+1}\right) \leq d_{\mathcal{H}}^{Z}\left(Z, \mathcal{P}_{n}\right)$, that is $R_{n+1} \leq R_{n}$.

2. $d_{Z}\left(p_{n+2}, \mathcal{P}_{n+1}\right) \leq d_{Z}\left(p_{n+1}, \mathcal{P}_{n}\right)$.

3. $s_{n}:=\min _{1 \leq i<j \leq n} d_{Z}\left(p_{i}, p_{j}\right) \geq \min _{1 \leq i<j \leq n+1} d_{Z}\left(p_{i}, p_{j}\right)=s_{n+1}$.

4. $s_{n}=d_{Z}\left(p_{n}, \mathcal{P}_{n-1}\right)$.

5. $d_{\mathcal{H}}^{Z}\left(Z, \mathcal{P}_{n}\right)=d_{Z}\left(p_{n+1}, \mathcal{P}_{n}\right)$.

6. $n \leq \frac{\mathbf{a}(Z)}{\inf _{z \in Z} \mathbf{a}\left(B_{Z}\left(z, \frac{s_{n}}{2}\right)\right)}$ where $\mathbf{a}$ is the area measure on $Z .^{15}$

These properties make it easy to compute $s_{n}$ and $r_{n}$ on the fly inside the algorithm, something useful when the objective is to to obtain either a pre-specified covering radius or a pre-specified separation.

Let us now assume that the discrete metric space $\left(\mathbb{Z}_{m}, d_{Z}\right)$ is a good random sampling of the underlying $\left(Z, d_{Z}\right)$ in the sense that $d_{\mathcal{H}}\left(Z, \mathbb{Z}_{m}\right) \leq r$ with a certain probability $p_{r, m}$, as discussed in Section $§ 3.3$. We then want to simplify $\mathbb{Z}_{m}$ in order to obtain a set $\mathcal{P}_{n}$ with $n$ points which is both a

\footnotetext{
${ }^{15}$ Note that with curvature bounds one can obtain a more explicit relationship between $s_{n}$ and $n$.
} 
good subsampling and a well separated covering net of $X .{ }^{16}$ We want to use our $n$ sampled points in the best possible way. We are then led to using the construction discussed above. For example, choose randomly one point $p_{1} \in \mathbb{Z}_{m}$ and consider $\mathcal{P}_{1}=\left\{p_{1}\right\}$. Run the procedure FPS until $n-1$ other points have been added to the set of points. ${ }^{17}$ Compute now $r_{n}:=\max _{q \in \mathbb{X}_{m}} d\left(q, \mathcal{P}_{n}\right)$. Then, also with probability $p_{r, m}, \mathcal{P}_{n}$ is a $\left(r+r_{n}\right)$-covering net of $X$ with separation $s_{n}$ as expressed in Lemma 3. Following this, we now use the notation $N_{Z, n}^{\left(\left(r+r_{n}\right), s_{n}\right)}$.

\subsection{Computing Geodesic Distances}

In our experiments we have always worked with submanifolds of $\mathbb{R}^{d}$. We have used a graph based distance computation following [29], or the exact distance, which can be computed only for certain examples (spheres, planes). We could also use the techniques developed for triangular surfaces in [33], or, being this the optimal candidate, the work on geodesics on (maybe noisy) point clouds developed in [39].

The geodesic computation leads to additional sources of (controllable) errors. We can not compute $d_{X}\left(x_{i}, x_{j}\right)$ and $d_{Y}\left(y_{i}, y_{j}\right)$ exactly, but rather approximate values $d_{X}^{h}\left(x_{i}, x_{j}\right)$ and $d_{Y}^{h^{\prime}}\left(y_{i}, y_{j}\right)$ for which error bounds are often available [39]. For some suitable function $f(\cdot, \cdot, \cdot, \cdot)$

$$
\left|d_{X}\left(x_{i}, x_{j}\right)-d_{X}^{h}\left(x_{i}, x_{j}\right)\right| \leq f(h, r, s, n)
$$

and

$$
\left|d_{Y}\left(y_{i}, y_{j}\right)-d_{Y}^{h^{\prime}}\left(y_{i}, y_{j}\right)\right| \leq f\left(h^{\prime}, r^{\prime}, s^{\prime}, n\right)
$$

where $h$ and $h^{\prime}$ control the degrees of approximation. These kind of bounds can be computed for all the approximations we have worked with (see [3], [33]), and also for methods like the one proposed in [39]. We omit in this report the inclusion of this source of errors in our considerations, results in that direction will reported elsewhere.

\subsection{Additional Implementation Details}

In this section we conclude the details on the implementation of the framework here proposed. The first step of the implementation is the computation of $d_{\mathcal{F}}$ and subsequently $\mathcal{L}$, which from the theory we described before, bounds the Gromov-Hausdorff distance.

We have implemented a simple algorithm. ${ }^{18}$ Considering the matrix of pairwise geodesic distances between points of $\mathbb{X}_{m}$, we need to determine whether there exists a submatrix of the whole distance matrix corresponding to $\mathbb{X}_{m}$ which has a small $d_{\mathcal{J}}$ distance to the corresponding interpoint distance matrix of a given $N_{Y, n}^{\left(R^{\prime}, s^{\prime}\right)}$. We select this latter covering net as the result of applying the FPS procedure to obtain a subsample consisting of $n$ points, where the first two points are selected to be at maximal distance from each other. To fix notation, let $\mathbb{X}_{m}=\left\{x_{1}, \ldots, x_{m}\right\}$ and $N_{Y, n}^{\left(R^{\prime}, s^{\prime}\right)}=\left\{y_{j_{1}}, \ldots, y_{j_{n}}\right\}$. We then use the following algorithm.

$$
(k=1,2) \text { Choose } x_{i_{1}} \text { and } x_{i_{2}} \text { such that }\left|d_{X}\left(x_{i_{1}}, x_{i_{2}}\right)-d_{Y}\left(y_{j_{1}}, y_{j_{2}}\right)\right| \text { is minimized. }
$$

\footnotetext{
${ }^{16}$ One more reason for wanting the subsampling to be well separated, besides the one given by Corollary 4 , is that intuitively, the more separated the covering net, the more efficient the use of the points to cover the metric space.

${ }^{17}$ As we mentioned before, the goal can be different: Keep adding points while the separation of the resulting subsampling is big enough as measured by some pre-specified constant $s>0$.

${ }^{18}$ This simpler algorithm in turn can be modified to be exhaustive and therefore rigorous, details will provided elsewhere.
} 
$(k>2)$ Let $x_{i_{k+1}} \in \mathbb{X}_{m}$ be such that $e_{k+1}\left(x_{i_{k+1}}\right)=\min _{1 \leq i_{l} \leq m} e_{k+1}\left(x_{i_{l}}\right)$ where

$$
e_{k+1}\left(x_{i_{l}}\right)=\max _{1 \leq r \leq k}\left|d_{X}\left(x_{i_{l}}, x_{i_{r}}\right)-d_{Y}\left(y_{j_{k+1}}, y_{j_{r}}\right)\right|
$$

We stop when $n$ points, $\left\{x_{i_{1}}, x_{i_{2}}, \ldots, x_{i_{n}}\right\}$ have been selected, and therefore a distance submatrix $\left(\left(d_{X}\left(x_{i_{u}}, x_{i_{v}}\right)\right)\right)_{u, v=1}^{n}$, is obtained.

Since we can write

$$
d_{\mathcal{J}}\left(\left\{x_{i_{1}}, \ldots, x_{i_{n}}\right\}, N_{Y, n}^{\left(R^{\prime}, s^{\prime}\right)}\right) \leq \frac{1}{2} \max _{1 \leq k \leq n} \max _{1 \leq t \leq k-1}\left|d_{X}\left(x_{i_{k}}, x_{i_{t}}\right)-d_{Y}\left(y_{j_{k}}, y_{j_{t}}\right)\right|=\frac{1}{2} \max _{1 \leq k \leq n} e_{k}\left(x_{i_{k}}\right)
$$

we then see that with our algorithm we are decreasing the error row-wise.

Of course, we now use the same algorithm to compute the other half of $d_{\mathcal{F}}$. We are currently studying computational improvements along with error bounds for the results provided by the algorithm.

\section{Examples}

We now present experiments that confirm the validity of the theoretical and computational framework introduced in previous sections. In the future, we plan to make these experiments more rigorous, including concepts of hypothesis testing. As a simplification, for our experiments we have only computed $d_{\mathcal{F}}$ neglecting the other term in $\mathcal{L}($ see $\S 3.1)$, which provides an estimate of the Gromov-Hausdorff proximity between the shapes.

We complemented the more complex data (as presented below) with simple shapes:

[Plane] $P_{\pi}=\left[-\frac{\pi}{\sqrt{8}}, \frac{\pi}{\sqrt{8}}\right]^{2}$ and $\mathbb{X}_{m}$ are points sampled uniformly from the square. Note that $\operatorname{diam}(X)=\pi$.

[Sphere] $S=\left\{x \in \mathbb{R}^{d}:\|x\|=1\right\}$ and $\mathbb{X}_{m}$ is a set of points uniformly distributed on the sphere. We generated the sample points using the method of Muller, see [42].

\subsection{Positive Detection}

We first test our framework when $X$ and $Y$ are isometric. We consider $X=Y$ and see whether we make the right decision based on the discrete measurements. Let $\mathbb{X}_{m}$ and $\mathbb{Y}_{m}$ be two independent sets composed of $m$ independent, uniformly distributed random points on $X$. We consider $X$ to be either the plane $P_{\pi}$ or the sphere $S$ as defined above. Given $n$, using the FPS procedure, we construct $N_{\mathbb{X}_{m}, n}$ and $N_{\mathbb{Y}_{m}, n}$ from $\mathbb{X}_{m}$ and $\mathbb{Y}_{m}$, respectively (we omit the supraindices since we won't use the values of covering radius and separation), and look for a metric match inside $\mathbb{X}_{m}$ and $\mathbb{Y}_{m}$, respectively, following the algorithm described in $\S 3.6$ for the computation of $d_{\mathcal{F}}\left(\mathbb{X}_{m}, \mathbb{Y}_{m}\right) \cdot{ }^{19}$ For each dataset we tested for values of $m \in M=\{500,600, \ldots, 2000\}$ and $n \in N=\{5,10,15, \ldots, 100\}$, and obtained the results reported below. In Tables 1 and 2 we show the values of $d_{\mathcal{F}}$ for selected values of $m$ and $n$. As expected, the values of $d_{\mathcal{F}}$ are small (see next section for the corresponding values when comparing non-isometric shapes). In Figure 1 we show a pseudocolor representation of the results for $d_{\mathcal{F}}$. In Figure 2 we plot the values of $d_{\mathcal{F}}$ for the two different random samplings of the sphere (reported in Table 2) against $m \in M$, see the caption for more details.

\footnotetext{
${ }^{19}$ Keep in mind that actually $d_{\mathcal{F}}\left(\mathbb{X}_{m}, \mathbb{Y}_{m}\right)$ depends on $n$, see its definition (12)
} 


\begin{tabular}{|c||c|c|c|c|c|c|c|c|}
\hline$n \backslash m$ & 500 & 700 & 900 & 1100 & 1300 & 1500 & 1700 & 1900 \\
\hline 5 & 0.036793 & 0.013088 & 0.015786 & 0.012885 & 0.036110 & 0.018160 & 0.016485 & 0.0074027 \\
15 & 0.043457 & 0.040605 & 0.043771 & 0.027194 & 0.028810 & 0.027367 & 0.032519 & 0.014247 \\
25 & 0.041845 & 0.044206 & 0.050095 & 0.036036 & 0.027655 & 0.026821 & 0.023060 & 0.031019 \\
35 & 0.061570 & 0.049158 & 0.031380 & 0.037598 & 0.030816 & 0.028623 & 0.033991 & 0.020764 \\
45 & 0.081975 & 0.056267 & 0.042198 & 0.055948 & 0.035188 & 0.038990 & 0.024451 & 0.036376 \\
55 & 0.087237 & 0.045492 & 0.063081 & 0.043192 & 0.045165 & 0.036363 & 0.033156 & 0.034592 \\
65 & 0.068935 & 0.049395 & 0.052482 & 0.046853 & 0.037285 & 0.035718 & 0.035569 & 0.031512 \\
75 & 0.070109 & 0.075802 & 0.051246 & 0.053371 & 0.045849 & 0.037692 & 0.044748 & 0.045059 \\
85 & 0.077863 & 0.053011 & 0.038660 & 0.046119 & 0.045688 & 0.036009 & 0.039273 & 0.036894 \\
95 & 0.077007 & 0.069984 & 0.059779 & 0.036691 & 0.035976 & 0.032634 & 0.035390 & 0.034774 \\
\hline
\end{tabular}

Table 1: Table with values of $d_{\mathcal{F}}$ for $X=P_{\pi}$ (a plane).

\begin{tabular}{|c||c|c|c|c|c|c|c|c|}
\hline$n \backslash m$ & 500 & 700 & 900 & 1100 & 1300 & 1500 & 1700 & 1900 \\
\hline 5 & 0.013282 & 0.025620 & 0.013855 & 0.024352 & 0.017305 & 0.010935 & 0.015408 & 0.013558 \\
15 & 0.057752 & 0.045298 & 0.049417 & 0.048648 & 0.034589 & 0.036678 & 0.028731 & 0.036313 \\
25 & 0.082785 & 0.062767 & 0.043617 & 0.045718 & 0.036738 & 0.033095 & 0.040189 & 0.033592 \\
35 & 0.077173 & 0.066819 & 0.059948 & 0.052525 & 0.035929 & 0.051900 & 0.049051 & 0.037428 \\
45 & 0.074482 & 0.069326 & 0.067096 & 0.054424 & 0.051452 & 0.057161 & 0.040566 & 0.040727 \\
55 & 0.089103 & 0.070744 & 0.066340 & 0.055915 & 0.061401 & 0.057970 & 0.055716 & 0.045899 \\
65 & 0.079456 & 0.087671 & 0.076762 & 0.067578 & 0.051363 & 0.049503 & 0.063492 & 0.043405 \\
75 & 0.082986 & 0.083790 & 0.069210 & 0.056734 & 0.060989 & 0.061746 & 0.048117 & 0.045362 \\
85 & 0.083577 & 0.075528 & 0.083344 & 0.067288 & 0.069203 & 0.058094 & 0.051962 & 0.054144 \\
95 & 0.10688 & 0.081448 & 0.071951 & 0.064473 & 0.060619 & 0.052794 & 0.053800 & 0.050700 \\
\hline
\end{tabular}

Table 2: Table with values of $d_{\mathcal{F}}$ for $X=S$ (a sphere).

\subsection{Positive Rejection}

We now proceed to compare shapes that are not isometric, starting with $X=P_{\pi}$ (a plane) and $Y=S$ (a sphere). In this case we expect to be able to detect, based on the finite point clouds, that $d_{\mathcal{F}}$ is large.

Table 3 and Figure 3 show the results of a simulation in which we compared the sphere $S$ and the plane $P_{\pi}$, while varying the covering net sizes and the total number of points uniformly sampled from them ( $n \in N$ and $m \in M$ as before). The experiments have been repeated 100 times to produce Table 3, and the reported values consist of the mean of these 100 tests. As expected, the values are larger than when comparing plane against plane or sphere against sphere.

\subsection{D-Shape Recognition of Articulated Objects}

We conclude the experiments with real (more complex) data. We have 4 sets of shapes, ${ }^{20}$ the crocodile, the giraffe, the hand and the body, each one with their corresponding bends. We ran the algorithm $N=6$ times with $n=70, m=2000$, using the 4 nearest neighbors graph to compute geodesic distances via isomap's engine. The data description and results are reported in Table

\footnotetext{
${ }^{20}$ The datasets were kindly provided to us by Prof. Kimmel and his group at the Technion.
} 

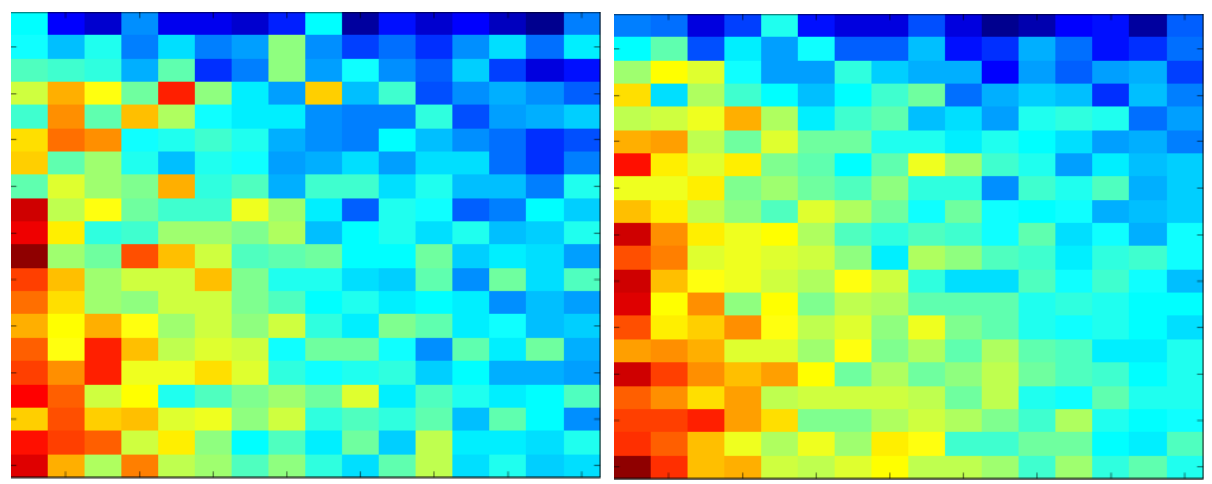

Figure 1: Graphic visualization of the results for the plane $P_{\pi}$ (on the left), and the sphere $S$ (on the right). Red corresponds to low values of $d_{\mathcal{F}}$ and blue larger values. On the horizontal axis, from left to right we have increasing values of $m$, while on the vertical axis, $n$ increases going upwards. Observe how the distance increases for fixed $m$ as $n$ increases in accordance with the fact that we have less freedom to choose the $n$ points from the given $m$. (This is a color figure.)

\begin{tabular}{|c||c|c|c|c|}
\hline$n \backslash m$ & 500 & 1000 & 1500 & 2000 \\
\hline 10 & $1.839 \times 10^{-1}$ & $1.902 \times 10^{-1}$ & $1.931 \times 10^{-1}$ & $1.942 \times 10^{-1}$ \\
25 & $1.834 \times 10^{-1}$ & $1.908 \times 10^{-1}$ & $1.920 \times 10^{-1}$ & $1.944 \times 10^{-1}$ \\
50 & $1.818 \times 10^{-1}$ & $1.899 \times 10^{-1}$ & $1.925 \times 10^{-1}$ & $1.933 \times 10^{-1}$ \\
75 & $1.873 \times 10^{-1}$ & $1.882 \times 10^{-1}$ & $1.936 \times 10^{-1}$ & $1.939 \times 10^{-1}$ \\
100 & $1.846 \times 10^{-1}$ & $1.913 \times 10^{-1}$ & $1.924 \times 10^{-1}$ & $1.936 \times 10^{-1}$ \\
\hline
\end{tabular}

Table 3: Values of $d_{\mathcal{F}}$ for a comparison between $P_{\pi}$ and $S$ for selected values of $n \in N$ and $m \in M$.

4. We note that not only is the technique able to discriminate between different objects but, as expected, it doesn't get confused by bends: The distances between a given object and the possible bends of another one are very similar, as it should be the case for isometry invariant recognition.

\section{Conclusions}

A theoretical and computational framework for comparing (smooth, connected and compact) submanifolds of $\mathbb{R}^{d}$ given as point clouds was introduced in this paper. The theoretical component is based on the Gromov-Hausdorff distance, which has been embedded in a probabilistic framework to deal with point clouds and computable discrete distances. Examples supporting this theory were provided.

The extension to more general metric spaces can be done, in principle, once one agrees upon some definition of uniform probability measure, something that could be done using the Hausdorff measure, which is defined from the metric.

Another related possible extension is that of admitting the points to be sampled from the manifolds with probability measures other than uniform. Actually, in the case of surfaces in $\mathbb{R}^{3}$ acquired by a $3 D$-Scanner, the probability measure models the acquisition process itself. In this case, the framework here presented can be extended for a wide family a probability measures, namely those which admit a density function which vanishes at most in sets of 0 -uniform measure, i.e. there are no holes in the acquisition process. In other situations it might simply make more 


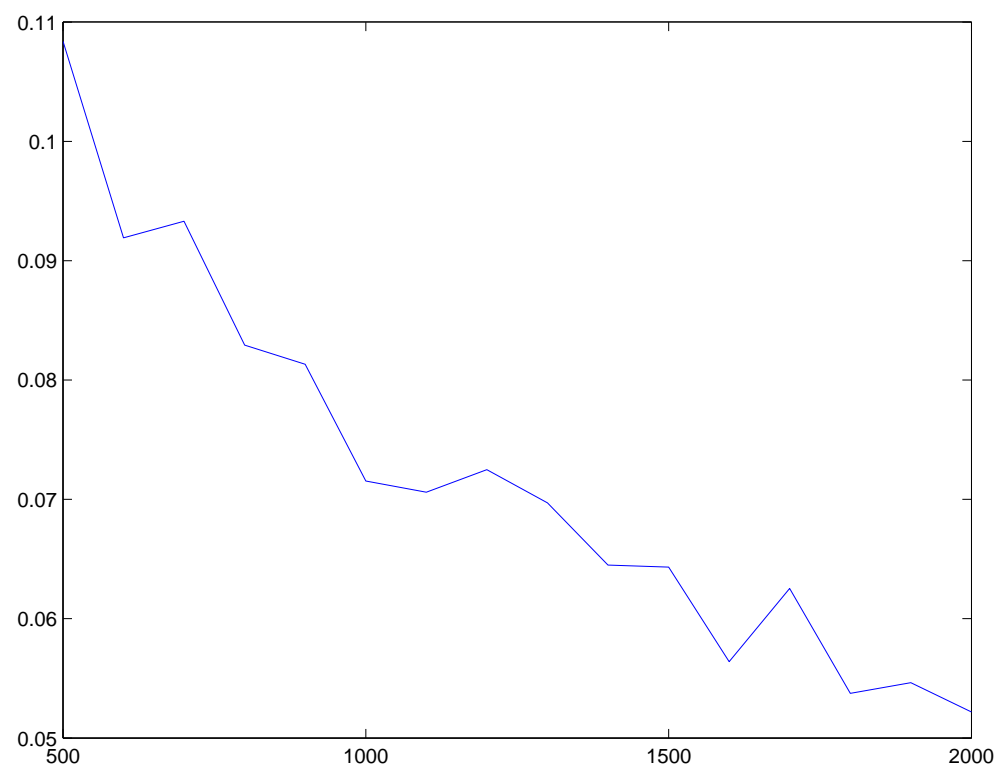

Figure 2: Plot of $\max _{n \in N} d_{\mathcal{F}}\left(\mathbb{X}_{m}, \mathbb{Y}_{m}\right)$ against $m$ for two different samplings of the sphere. It is based on the data reported in Table 2.

sense to consider the recognition problem for triplets $(X, d, \mu)$, where $(X, d)$ is a metric space and $\mu$ is a (probability) measure defined on sets of $X$.

An interesting extension which might make the computational analysis easier would be working with other definitions of Hausdorff distance. For example, remembering that the Hausdorff distance between $X, Y \subset Z,((Z, d, \mu)$ a metric space with probability measure $\mu)$ is defined as

$$
d_{\mathcal{H}}^{Z}(X, Y):=\max \left(\sup _{x \in X} d(x, Y), \sup _{y \in Y} d(y, X)\right) .
$$

Then, one can consider substituting each of the supremum inside the $\max ($,$) by an L_{p}$-approximation (for $p \geq 1$ ), for example: $\sup _{x \in X} d(x, Y) \leftrightarrow\left(\int d^{p}(x, Y) \mu(d x)\right)^{1 / p}$, and similarly for the other supremum to obtain, also allowing for a $L_{q}$-approximation of the $\max (q \geq 1)$ :

$$
d_{\mathcal{H}_{p, q}}^{Z}(X, Y):=\left(\left(\int d^{p}(x, Y) \mu(d x)\right)^{q / p}+\left(\int d^{p}(y, X) \mu(d y)\right)^{q / p}\right)^{1 / q}
$$

The corresponding notion of $(p, q)$-Gromov-Hausdorff distance is then defined. In particular, it would be interesting to derive the $(p, q)$ version of Property 5 of Proposition 1.

We are currently working on proving the correctness of the algorithm described in $\S 3.6$, improving its computational efficiency, performing additional experiments adding hypotheses testing techniques, and in particular, comparing high dimensional point clouds with data from image sciences and neuroscience. These further results and extensions will be reported elsewhere.

\section{Acknowledgments}

We thank Prof. Omar Gil, Prof. Ron Kimmel and Prof. Ofer Zeitouni for stimulating conversations on the subject of this paper. A. Elad and R. Kimmel provided valuable data for the experiments. 

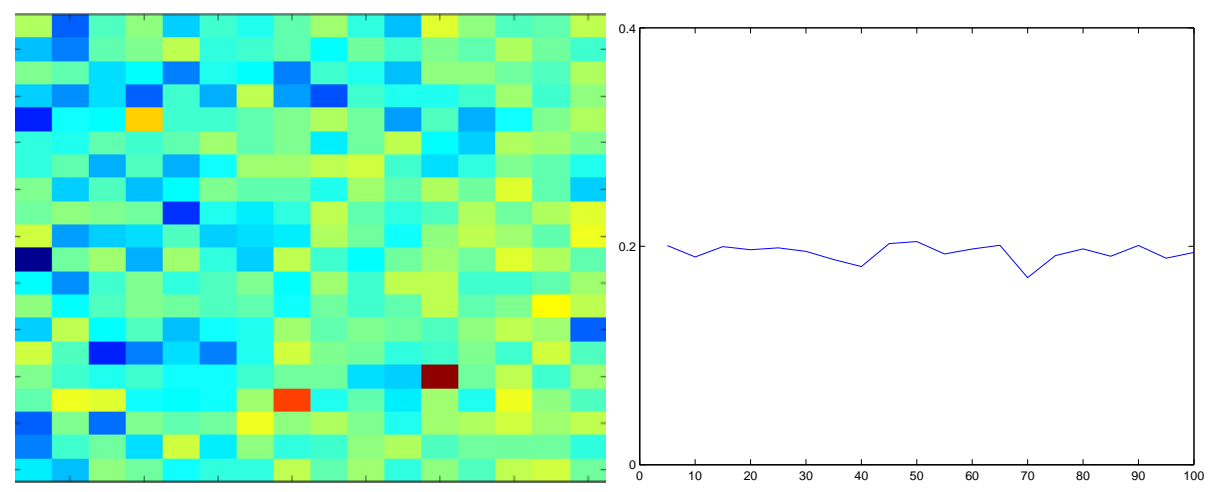

Figure 3: Left: Graphic visualization of the results for the comparison between the plane $P_{\pi}$ and the Sphere $S$. Red corresponds to low values of $d_{\mathcal{F}}$ and blue larger values. On the horizontal axis, from left to right we find increasing values of $m$, and on the vertical axis, $n$ increases going upwards. Right: Plot of the values of $d_{\mathcal{F}}$ obtained against $n$, the size of the FPS covering net, for fixed $m=2000$. (This is a color figure.)

This work is supported in part by the Office of Naval Research, the National Science Foundation, and the National Institutes of Health. The work of FM is partly supported by CSIC-Uruguay.

\section{References}

[1] E. Arias-Castro, D. Donoho, and X. Huo, "Near-optimal detection of geometric objects by fast multiscale methods," Stanford Statistics Department TR, 2003.

[2] M. Belkin and P. Niyogi, "Laplacian eigenmaps for dimensionality reduction and data representation," University of Chicago CS TR-2002-01, 2002.

[3] M. Bernstein, V. de Silva, J. Langford, and J. Tenenbaum, "Graph approximations to geodesics on embedded manifolds," http://isomap.stanford.edu/BdSLT.ps

[4] J. Borwein and O. Hijab. http://www.siam.org/journals/problems/downloadfiles/99-5sii.pdf

[5] J-D. Boissonnat and F. Cazals, "Coarse-to-fine surface simplification with geometric guarantees," in A. Chalmers and T-M. Rhyne, Editors, EUROGRAPHICS '01, Manchester, 2001.

[6] M. Botsch, A. Wiratanaya, and L. Kobbelt, "Efficient high quality rendering of point sampled geometry," EUROGRAPHICS Workshop on Rendering, 2002.

[7] M. Boutin and G. Kemper "On reconstructing n-point configurations from the distribution of distances or areas," Adv. Appl. Math. 32(4), pp. 625-825.

[8] A. Bronstein, M. Bronstein, A. Spira and Ron Kimmel "Face recognition from facial surface metric," Proceedings of ECCV 2004 (Part II), pp. 225-237.

[9] D. Burago, Y. Burago, and S. Ivanov, A Course in Metric Geometry, AMS Graduate Studies in Mathematics, Vol. 33.

[10] D. Burago and B. Kleiner "Separated nets in Euclidean space and Jacobians of bi-Lipschitz maps," Geom. Funct. Anal. 8, pp. 273-282, 1998. 
[11] D. Burago and B. Kleiner, "Rectifying separated nets," Geom. Funct. Anal. 12, pp 80-92, 2002.

[12] G. Carlsson, A. Collins, L. Guibas, and A. Zomorodian, "Persistent homology and shape description I, preprint, Stanford Math Department, September 2003.

[13] G. Charpiat, O. Faugeras, and R. Keriven. "Shape metrics, warping, and statistics, Proceedings of the International Conference on Image Processing, IEEE Signal Processing Society, September 2003.

[14] T. K. Dey, J. Giesen, and J. Hudson, "Decimating samples for mesh simplification," Proc. 13th Canadian Conference on Computational Geometry, pp. 85-88, 2001.

[15] D. L. Donoho and C. Grimes, "When does ISOMAP recover the natural parametrization of families of articulated images?," Technical Report 2002-27: Department of Statistics, Stanford University, 2002.

[16] D. L. Donoho and C. Grimes, "Hessian eigenmaps: New locally-linear embedding techniques for high-dimensional data," Technical Report Department of Statistics, Stanford University, 2003 .

[17] N. Dyn, M. S. Floater, and A. Iske, "Adaptive thinning for bivariate scattered data," Journal of Computational and Applied Mathematics 145(2), pp. 505-517, 2002.

[18] A. Elad (Elbaz) and R. Kimmel, "Bending invariant representations for surfaces," In Proc. of CVPR'01, Hawaii, Dec. 2001.

[19] W. Feller, An Introduction to Probability Theory and its Applications, John Wiley \& Sons, Inc., New York-London-Sydney, 1971.

[20] M. S. Floater and A. Iske, "Thinning algorithms for scattered data interpolation," BIT Numerical Mathematics 38, pp. 705-720, 1998.

[21] J. Giesen and U, Wagner, "Shape dimension and intrinsic metric from samples of manifolds with high co-dimension," ACM Symposium on Computational Geometry, San Diego, June 2003.

[22] M.T. Goodrich, J.S.B. Mitchell and M.W. Orletsky, "Approximate geometric pattern matching under rigid motions," IEEE Transactions on Pattern Analysis and Machine Intelligence, 21:4, pp. $371-379,1999$.

[23] A. Gray, Tubes, Addison Wesley, 1990.

[24] M. Gromov, Metric Structures for Riemannian and Non-Riemannian Spaces, Progress in Mathematics 152, Birkhäuser Boston, Inc., Boston, MA, 1999.

[25] M. Gromov, Geometric group theory, Vol. 2: Asymptotic invariants of infinite groups. Edited by A. Niblo and Martin A. Roller. London Math. Soc. Lecture Note Ser., vol. 182, Cambridge Univ. Press, 1993.

[26] M. Gross et al, Point Based Computer Graphics, EUROGRAPHICS Lecture Notes, 2002 (graphics.stanford.edu/ niloy/research/papers/ETH/PointBasedComputerGraphics_TutorialNotes.pdf). 
[27] K. Grove, "Metric differential geometry," Differential geometry (Lyngby, 1985), 171-227, Lecture Notes in Math., 1263, Springer, Berlin, 1987.

[28] D. P. Huttenlocher, G. A. Klanderman and W. J. Rucklidge "Comparing images using the Hausdorff distance," IEEE Transactions on Pattern Analysis and Machine Intelligence, 15:9, 1993.

[29] http://isomap.stanford.edu

[30] P. W. Jones, "Rectifable sets and the traveling salesman problem," Invent. Math. 102, pp. $1-15,1990$.

[31] D. W. Kahn, Topology. An Introduction to the Point-Set and Algebraic Areas, Williams \& Wilkins Co., Baltimore, Md., 1975.

[32] N. J. Kalton and M. I. Ostrovskii, "Distances between Banach spaces," Forum Math. 11:1, pp. 17-48, 1999.

[33] R. Kimmel and J. A. Sethian, "Computing geodesic paths on manifolds," Proc. National Academy of Sciences 95:15, pp. 8431-8435, 1998.

[34] G. Leibon and D. Letscher, "Delaunay triangulations and Voronoi diagrams for Riemannian manifolds," Computational Geometry 2000, Hong Kong, 2000.

[35] G. Lerman, "How to partition a low-dimensional data set into disjoint clusters of different geometric structure," preprint, 2000.

[36] L. Linsen, "Point cloud representation," CS Technical Report, University of Karlsruhe, 2001.

[37] L. Linsen and H. Prautzsch, "Local versus global triangulations," EUROGRAPHICS '01, 2001.

[38] C.T. McMullen "Lipschitz maps and nets in Euclidean space," Geom. Funct. Anal. 8, pp. 304-314, 1998.

[39] F. Mémoli and G. Sapiro, "Distance functions and geodesics on point clouds," IEEE Workshop on Variational and Level-Sets Methods in Computer Vision, Nice, France, October 2003. Also as IMA Report 1925 (www.ima.umn.edu), May 2003.

[40] N. J. Mitra and A. Nguyen, "Estimating surface normals in noisy point cloud data," $A C M$ Symposium on Computational Geometry, San Diego, June 2003.

[41] C. Moenning and N. A. Dodgson, "Fast marching farthest point sampling for implicit surfaces and point clouds," Computer Laboratory Technical Report 565, available online at http://www.cl.cam.ac.uk/users/cm230/docs/pdfs/FastFPSISPC.pdf.

[42] E. W. Weisstein. "Hypersphere point picking," From MathWorld-A Wolfram Web Resource. http://mathworld.wolfram.com/HyperspherePointPicking.html

[43] V.V. Nekrashevych, "On equivalence of nets in hyperbolic spaces," Dopov. Nats. Akad. Nauk Ukr. Mat. Prirodozn. Tekh. Nauki, pp. 18-21, 1997.

[44] M. Pauly and M. Gross, "Spectral processing of point-sampled geometry," ACM SIGGRAPH, pp. 379-386, 2001 
[45] M. Pauly, M. Gross, and L. Kobbelt, "Efficient simplification of point-sampled surfaces," IEEE Visualization 2002.

[46] P. Petersen, Riemannian Geometry, Springer-Verlag, New York, 1998.

[47] P. Petersen, "Gromov-Hausdorff convergence of metric spaces," Differential geometry: Riemannian geometry (Los Angeles, CA, 1990), pp 489-504, Proc. Sympos. Pure Math., 54, Part 3, Amer. Math. Soc., Providence, RI, 1993.

[48] S. Rusinkiewicz and M. Levoy, "QSplat: A multiresolution point rendering system for large meshes," Computer Graphics (SIGGRAPH 2000 Proceedings), 2000.

[49] J. B. Tenenbaum, V. de Silva, and J. C. Langford, "A global geometric framework for nonlinear dimensionality reduction," Science, pp. 2319-2323, December 2000.

[50] D.Toledo. Journal: Bull. Amer. Math. Soc. 33 (1996), 395-398.

[51] H. Von Schelling, "Coupon collecting for unequal probabilities," Amer. Math. Monthly 61, pp. 306-311, 1954.

[52] M. Zwicker, M. Pauly, O. Knoll, and M. Gross, "PointShop 3D: An interactive system for point-based surface editing," SIGGRAPH, 2002. 


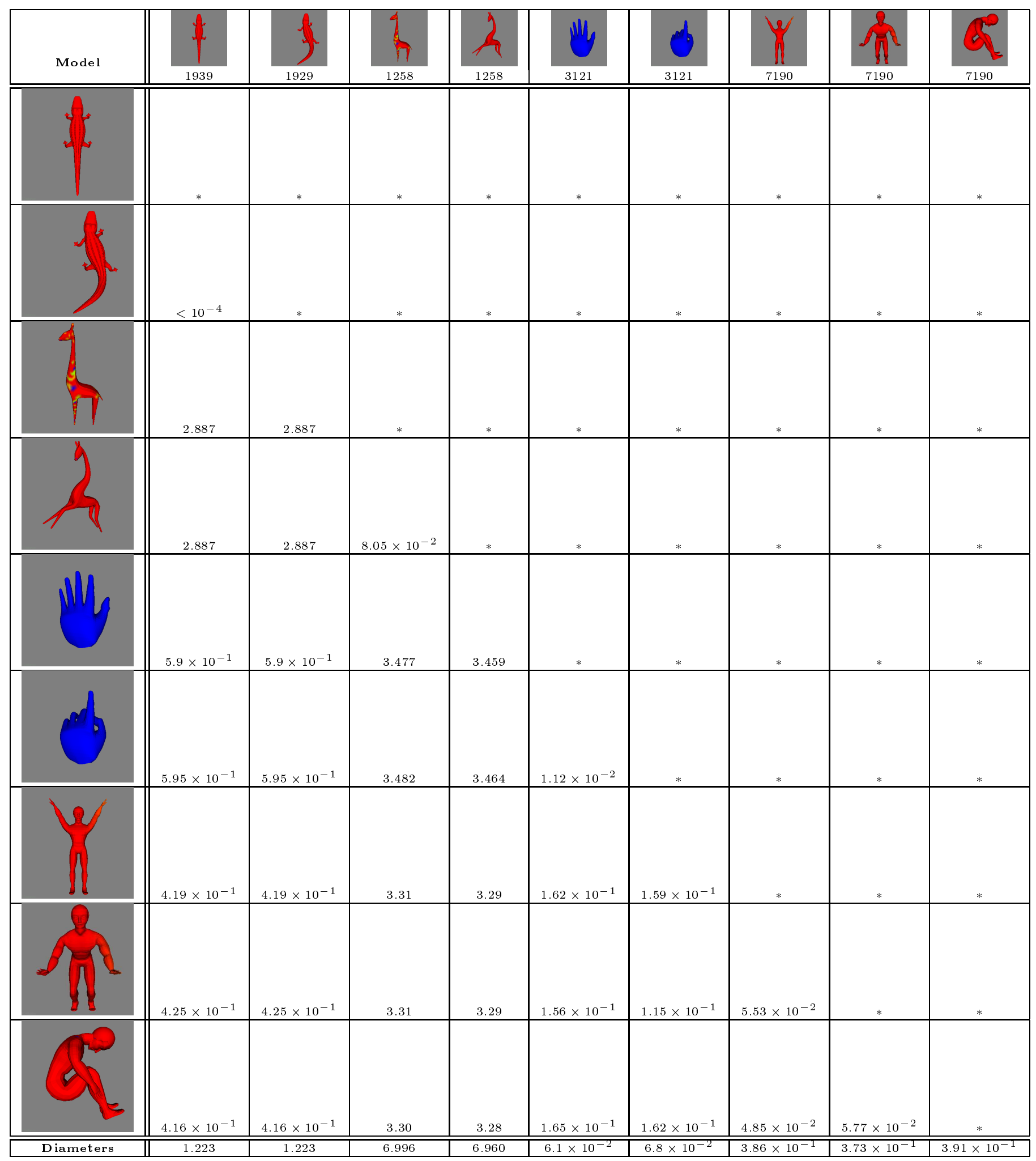

Figure 4: Comparison results for the complex objects described in $\S 4.3$. The number of points per model are indicated in the first row under the corresponding figure. 